\title{
FULLY-DISCRETE FINITE ELEMENT NUMERICAL SCHEME WITH DECOUPLING STRUCTURE AND ENERGY STABILITY FOR THE CAHN-HILLIARD PHASE-FIELD MODEL OF TWO-PHASE INCOMPRESSIBLE FLOW SYSTEM WITH VARIABLE DENSITY AND VISCOSITY
}

\author{
Chuanjun Chen $^{1}$ and Xiaofeng Yang ${ }^{2, *}$
}

\begin{abstract}
We construct a fully-discrete finite element numerical scheme for the Cahn-Hilliard phasefield model of the two-phase incompressible flow system with variable density and viscosity. The scheme is linear, decoupled, and unconditionally energy stable. Its key idea is to combine the penalty method of the Navier-Stokes equations with the Strang operator splitting method, and introduce several nonlocal variables and their ordinary differential equations to process coupled nonlinear terms. The scheme is highly efficient and it only needs to solve a series of completely independent linear elliptic equations at each time step, in which the Cahn-Hilliard equation and the pressure Poisson equation only have constant coefficients. We rigorously prove the unconditional energy stability and solvability of the scheme and carry out numerous accuracy/stability examples and various benchmark numerical simulations in $2 \mathrm{D}$ and $3 \mathrm{D}$, including the Rayleigh-Taylor instability and rising/coalescence dynamics of bubbles to demonstrate the effectiveness of the scheme, numerically.
\end{abstract}

Mathematics Subject Classification. 65N12, 65M12, 65M70.

Received February 17, 2021. Accepted September 2, 2021.

\section{INTRODUCTION}

When the Cahn-Hilliard-Navier-Stokes model (CH-NS, for short) of a two-phase incompressible flow system is coupled with variable density and viscosity, the existence of the variable density will cause many complicated terms to appear in the coupled system consisting of the Cahn-Hilliard equation and the incompressible NavierStokes equation. Therefore, although this model can simulate many interesting fluid interface phenomena very effectively, cf. [1, 13, 16, 22, 25,32], how to construct an effective fully discrete numerical scheme for such a highly complex coupled nonlinear system, making it not only linear, fully decoupled, but also capable of owning unconditional energy stability has always been a very challenging problem.

We know that there exist many very successful attempts to construct effective numerical schemes for the two equations that compose the two-component flow-coupled phase-field model, namely, the vari-

Keywords and phrases. Variable density, fully-decoupled, phase-field, Cahn-Hilliard, Navier-Stokes, unconditional energy stability.

1 School of Mathematics and Information Sciences, Yantai University, Yantai 264005, P.R. China.

2 Department of Mathematics, University of South Carolina, Columbia, SC 29208, USA.

* Corresponding author: xfyang@math.sc.edu. 
able density fluid equation and the Cahn-Hilliard equation. Efficient schemes include the so-called projection/gauge/penalty methods (see [17, 19, 20,28, 29,32]) for solving the incompressible Navier-Stokes equations with constant/variable density and viscosity, the linear stabilization, and the convex splitting/Invariant Energy Quadratization (IEQ) and its various version called Scalar Auxiliary Variable (SAV)/nonlinear functional derivative/nonlinear quadrature/Exponential Time Differencing (ETD) for solving the Cahn-Hilliard equation, see $[7,8,12,15,21,23,30,31,33,35,42,44-46]$ and the references therein. Therefore, a natural question may arise, whether a simple combination of these successful schemes can obtain the expected effective numerical scheme with certain characteristics, such as complete decoupling, linearity, easy-implementation, and unconditional energy stability.

However, the fact is that for the variable density model, as far as the author knows, the only numerical technique that can effectively handle the coupling of flow field and phase-field variable and meet the requirements of owning a fully decoupled structure and unconditional energy stability is the stabilization technique developed in $[4,25,33]$. However, the stabilization technique also has some obvious disadvantages. For example, one has to solve the Cahn-Hilliard equations with variable coefficients (related to $1 / \rho$ ) at each time step due to the applied stabilization term. This inevitably leads to a higher computational cost than solving a constant-coefficient system. Moreover, in the momentum equation, the scheme developed in $[4,25,33]$ linearizes the advection terms implicitly, which leads to further calculation costs in practice (see Rem. 3.7 for details and Fig. 2c for computational efficiency).

Therefore, on the basis of the scheme developed in $[4,25,33]$, for the flow-coupled two-phase Cahn-Hilliard model with variable density and viscosity, we aim to construct a fully discrete finite element scheme to overcome the shortcomings of stabilization technique. More precisely, we expect that the resulting scheme can solve as many constant coefficient systems as possible, so as to reduce the computational cost as much as possible while maintaining linearity, and owning a decoupling structure and unconditional energy stability. To this end, we note that many nonlinear coupling items satisfy the so-called "zero-energy-contribution" feature (see Rem. 2.1). By combining this particular property with the operator splitting method, we introduce several nonlocal variables and design some special ordinary differential equations (ODE). These variables and ODEs play a vital role in obtaining unconditional energy stability and the decoupling structure of the numerical scheme. We further combine this new decoupling technique with several other efficient approaches, including the penalty method for the fluid momentum equation, the quadratization method for the nonlinear energy potential to arrive at a numerical scheme with the desired properties. The actual implementation of the scheme is also very simple. The calculation of each time step only includes solving several elliptic equations, among which the Cahn-Hilliard equation has constant coefficients, the fluid momentum equation has only two variable coefficient terms, and the pressure Poisson equation also has constant coefficients. The solvability and unconditional energy stability of the proposed scheme are given, and various $2 \mathrm{D}$ and $3 \mathrm{D}$ numerical examples are further simulated, including Rayleigh-Taylor instability and liquid bubble rising dynamics, to numerically demonstrate the stability and accuracy of the scheme.

We organize the rest of the article as follows. In Section 2, we introduce the variable density/viscosity twophase flow-coupled Cahn-Hilliard model. In Section 3, a numerical scheme is constructed and its unconditional energy stability is strictly proved. The detailed implementation process as well as the solvability of each step are also given. In Section 4, we carry out numerical examples to verify the stability and accuracy. In Section 5 , some concluding remarks are given.

\section{The MODEL AND ITS ENERGy STRUCTURE}

The CH-NS model with variable density and viscosity for the two-phase fluid flow system was developed in [1]. Before introducing it, some notations are given here. The $L^{2}$ inner product of any two functions $\phi(\boldsymbol{x})$ and $\psi(\boldsymbol{x})$ is denoted by $(\phi, \psi)=\int_{\Omega} \phi(\boldsymbol{x}) \psi(\boldsymbol{x}) \mathrm{d} \boldsymbol{x}$, and the $L^{2}$ norm of $\phi(\boldsymbol{x})$ is denoted by $\|\phi\|=(\phi, \phi)^{\frac{1}{2}}$.

Suppose that the computational domain $\Omega \in \mathbb{R}^{\operatorname{dim}} \operatorname{dim}=2,3$ is a smooth, open bounded and connected domain. To represent a two-phase immiscible fluid mixture, a phase-field variable $\phi(\boldsymbol{x}, t)$ is introduced such 
that:

$$
\phi(x, t)= \begin{cases}1 & \text { fluid 1, } \\ -1 & \text { fluid 2, }\end{cases}
$$

with a thin, smooth transition region with the width $O(\epsilon)(\epsilon \ll 1)$ connecting these two distinct values. Note that $\int_{\Omega} \frac{1+\phi}{2} \mathrm{~d} \boldsymbol{x}$ and $\int_{\Omega} \frac{1-\phi}{2} \mathrm{~d} \boldsymbol{x}$ can also be viewed as the volume fraction of the fluid 1 and 2 , respectively. The density and the viscosity of fluid 1 and 2 are set as $\rho_{1}, \nu_{1}$ and $\rho_{2}, \nu_{2}$, respectively. We assume $\rho_{1} \leq \rho_{2}$ without loss of generality. Then, the governing PDE system of the variable density/viscosity two-phase system constructed in [1] reads as:

$$
\begin{aligned}
\phi_{t}+\nabla \cdot(\mathbf{u} \phi) & =M \Delta \mu, \\
\mu & =\lambda\left(-\epsilon \Delta \phi+\frac{1}{\epsilon} f(\phi)\right), \\
\rho(\phi)\left(\mathbf{u}_{t}+(\mathbf{u} \cdot \nabla) \mathbf{u}\right)+\boldsymbol{J}(\mu) \cdot \nabla \mathbf{u}-\nabla \cdot(\nu(\phi) D(\mathbf{u}))+\nabla p+\phi \nabla \mu & =0, \\
\nabla \cdot \mathbf{u} & =0,
\end{aligned}
$$

where

$$
\begin{aligned}
D(\mathbf{u}) & =\nabla \mathbf{u}+(\nabla \mathbf{u})^{T}, \\
\boldsymbol{J}(\mu) & =M \frac{\rho_{2}-\rho_{1}}{2} \nabla \mu, \\
\rho(\phi) & =\frac{\rho_{1}-\rho_{2}}{2} \phi+\frac{\rho_{1}+\rho_{2}}{2}, \\
\nu(\phi) & =\frac{\nu_{1}-\nu_{2}}{2} \phi+\frac{\nu_{1}+\nu_{2}}{2} .
\end{aligned}
$$

The the initial conditions read as

$$
\left.\mathbf{u}\right|_{(t=0)}=\mathbf{u}^{0},\left.p\right|_{(t=0)}=p^{0},\left.\phi\right|_{(t=0)}=\phi^{0},
$$

and the boundary conditions read as

$$
\left.\mathbf{u}\right|_{\partial \Omega}=\mathbf{0},\left.\partial_{\mathbf{n}} \phi\right|_{\partial \Omega}=\left.\partial_{\mathbf{n}} \mu\right|_{\partial \Omega}=0 .
$$

Here, $\mathbf{u}$ is the fluid velocity, $p$ is the pressure, $\rho(\phi)$ is the density, and $\nu(\phi)$ is the viscosity, $\mu$ is the chemical potential, $\lambda$ is the surface tension parameter, $f(\phi)=\phi^{3}-\phi$ is the derivative of the nonlinear double-well potential $F(\phi)=\frac{1}{4}\left(\phi^{2}-1\right)^{2}$, and $\mathbf{n}$ is the unit outward normal on the boundary $\partial \Omega$. The nonlinear term $\phi \nabla \mu$ is the surface tension term that is derived from the stress tensor, $(\mathbf{u} \cdot \nabla) \mathbf{u}$ and $\nabla \cdot(\mathbf{u} \phi)$ are two fluid advection terms. Note that periodic boundary conditions are another commonly used boundary condition.

The governing system (2.2)-(2.5) holds the law of energy dissipation, which can be derived by performing standard energy estimate and shown by the following theorem.

Theorem 2.1. The system (2.2)-(2.5) holds

$$
\frac{\mathrm{d}}{\mathrm{d} t} E(\rho, \mathbf{u}, \phi)=-M\|\nabla \mu\|^{2}-\frac{1}{2} \int_{\Omega} \nu|D(\mathbf{u})|^{2} \mathrm{~d} \boldsymbol{x} \leq 0,
$$

where

$$
E(\rho, \mathbf{u}, \phi)=\int_{\Omega}\left(\frac{1}{2} \rho|\mathbf{u}|^{2}+\lambda \frac{\epsilon}{2}|\nabla \phi|^{2}+\frac{\lambda}{\epsilon} F(\phi)\right) \mathrm{d} \boldsymbol{x}
$$

is the total free energy of the system. 
Proof. First, we multiply the $L^{2}$ inner product of (2.2) with $\mu$, of $(2.3)$ with $-\phi_{t}$, and perform integration by parts to get

$$
\begin{aligned}
\left(\phi_{t}, \mu\right) & =-\underbrace{(\nabla \cdot(\mathbf{u} \phi), \mu)}_{\mathrm{I}}-M\|\nabla \mu\|^{2}, \\
-\left(\mu, \phi_{t}\right)+\lambda \frac{\mathrm{d}}{\mathrm{d} t} \int_{\Omega}\left(\frac{\epsilon}{2}|\nabla \phi|^{2}+\frac{1}{\epsilon} F(\phi)\right) \mathrm{d} \boldsymbol{x} & =0 .
\end{aligned}
$$

Second, we mulitply the $L^{2}$ inner product of (2.4) with $\mathbf{u}$, use integration by parts, and apply the divergence-free condition (2.5) to obtain

$$
\left(\rho \mathbf{u}_{t}, \mathbf{u}\right)=-\frac{1}{2} \int_{\Omega} \nu|D(\mathbf{u})|^{2} \mathrm{~d} \boldsymbol{x}-\underbrace{(\phi \nabla \mu, \mathbf{u})}_{\text {II }}-\underbrace{(\rho(\mathbf{u} \cdot \nabla) \mathbf{u}, \mathbf{u})}_{\text {III }}-\underbrace{(\boldsymbol{J} \cdot \nabla \mathbf{u}, \mathbf{u})}_{\mathrm{V}} .
$$

Third, from (2.2), (2.7) and (2.8), we obtain

$$
\rho_{t}+\nabla \cdot(\rho \mathbf{u})+\nabla \cdot \boldsymbol{J}=0
$$

By multiplying $\frac{1}{2} \mathbf{u}$ to $(2.17)$, we derive

$$
\frac{1}{2} \rho_{t} \mathbf{u}=-\frac{1}{2} \nabla \cdot(\rho \mathbf{u}) \mathbf{u}-\frac{1}{2} \nabla \cdot \boldsymbol{J} \mathbf{u} .
$$

Hence, we take the $L^{2}$ inner product of (2.18) with $\mathbf{u}$ to derive

$$
\left(\rho_{t}, \frac{1}{2}|\mathbf{u}|^{2}\right)=-\underbrace{\frac{1}{2}(\nabla \cdot(\rho \mathbf{u}) \mathbf{u}, \mathbf{u})}_{\text {IV }}-\underbrace{\frac{1}{2}(\nabla \cdot \boldsymbol{J} \mathbf{u}, \mathbf{u})}_{\text {VI }} .
$$

Finally, by combining (2.14)-(2.16), and (2.19), and using Remark 2.1, we get (2.12).

Remark 2.1. During the process of deriving (2.12), many nonlinear terms are canceled due to integration by parts and the boundary condition $\left.\mathbf{u}\right|_{\partial \Omega}=0$, including

$$
\begin{array}{ll}
\text { I and II : } & (\nabla \cdot(\mathbf{u} \phi), \mu)+(\phi \nabla \mu, \mathbf{u})=0, \\
\text { III and IV : } & (\rho(\mathbf{u} \cdot \nabla) \mathbf{u}, \mathbf{u})+\frac{1}{2}(\nabla \cdot(\rho \mathbf{u}) \mathbf{u}, \mathbf{u})=0, \\
\text { V and VI : } & (\boldsymbol{J} \cdot \nabla \mathbf{u}, \mathbf{u})+\frac{1}{2}(\nabla \cdot \boldsymbol{J} \mathbf{u}, \mathbf{u})=0 .
\end{array}
$$

Equations (2.20)-(2.22) can be regarded as the "zero-energy-contribution" property [36-41], namely, the contribution of all these nonlinear terms to the total free energy of the system is zero. We will use this property to design decoupling type numerical schemes in the next section.

\section{NUMERICAL SCHEME}

We expect to construct an effective time marching scheme that can own the desired properties of decoupling, linearity, and unconditional energy stability. To overcome the challenges on how to discretize the nonlinear terms to obtain the energy stability, we design several nonlocal variables and their associated ODEs and reformulate the PDE system to an equivalent one. 


\subsection{Reformation to an equivalent PDE system}

First, we introduce a nonlocal scalar variable $U(t)$, which is defined as

$$
U(t)=\sqrt{\int_{\Omega}\left(F(\phi)-\frac{s}{2} \phi^{2}\right) \mathrm{d} \boldsymbol{x}+B}=\sqrt{\int_{\Omega}\left(\frac{\left(\phi^{2}-1\right)^{2}}{4}-\frac{s}{2} \phi^{2}\right) \mathrm{d} \boldsymbol{x}+B},
$$

where $s$ and $B$ are two positive constants. The new variable $U$ is defined as the square root of the nonlinear double-well potential after extracting a quadratic term $\frac{s}{2} \phi^{2}$. This quadratic extraction term can help to maintain the $H^{1}$ stability of $\phi$ (see [43]). The constant $B$ is used to ensure the radicand positive since $F(\phi)-\frac{s}{2} \phi^{2}$ is always bounded from below. The use of a new variable $U$ to "quadratize" the nonlinear energy potential is the so-called IEQ or SAV method, see [8], which is an efficient method to obtain the linear and stable scheme for gradient flow type models.

Second, we introduce two nonlocal variables $Q(t)$ and $R(t)$ and design two special ODEs for them that read as

$$
\left\{\begin{array}{l}
Q_{t}=(\nabla \cdot(\mathbf{u} \phi), \mu)+(\phi \nabla \mu, \mathbf{u}), \\
\left.Q\right|_{(t=0)}=1
\end{array}\right.
$$

and

$$
\left\{\begin{array}{l}
R_{t}=(\rho(\mathbf{u} \cdot \nabla) \mathbf{u}, \mathbf{u})+\frac{1}{2}(\nabla \cdot(\rho \mathbf{u}) \mathbf{u}, \mathbf{u})+(\boldsymbol{J} \cdot \nabla \mathbf{u}, \mathbf{u})+\frac{1}{2}(\nabla \cdot \boldsymbol{J} \mathbf{u}, \mathbf{u}), \\
\left.R\right|_{(t=0)}=1,
\end{array}\right.
$$

where $\left.\mathbf{u}\right|_{\partial \Omega}=0$. Note that the design of these ODEs is due the nonlinear terms mentioned in Remark 2.1 satisfying the so-called zero-energy-contribution property. By using the initial conditions and Remark 2.1, it is easy to see that these two ODEs are equivalent to $Q_{t}=0$ and $R_{t}=0$. Thus from their initial conditions, it is easy to see that $Q(t)=1$ and $R(t)=1$ are their exact solutions.

Then, using the new variables $Q, R, U$, the CH-NS system $(2.2)-(2.5)$ can be rewritten to the following equivalent form:

$$
\begin{aligned}
\phi_{t}+Q \nabla \cdot(\mathbf{u} \phi) & =M \Delta \mu \\
\mu & =\lambda\left(-\epsilon \Delta \phi+\frac{s}{\epsilon} \phi+\frac{1}{\epsilon} H U\right) \\
\rho \mathbf{u}_{t}+\frac{1}{2} \rho_{t} \mathbf{u}-\nabla \cdot(\nu D(\mathbf{u})) & +\nabla p+Q \phi \nabla \mu+R \rho(\mathbf{u} \cdot \nabla) \mathbf{u}+\frac{1}{2} R \nabla \cdot(\rho \mathbf{u}) \mathbf{u}+R \boldsymbol{J} \cdot \nabla \mathbf{u}+\frac{1}{2} R \nabla \cdot \boldsymbol{J} \mathbf{u}=0 \\
\nabla \cdot \mathbf{u} & =0 \\
U_{t} & =\frac{1}{2}\left(H, \phi_{t}\right) \\
Q_{t} & =(\nabla \cdot(\mathbf{u} \phi), \mu)+(\phi \nabla \mu, \mathbf{u}), \\
R_{t} & =(\rho(\mathbf{u} \cdot \nabla) \mathbf{u}, \mathbf{u})+\frac{1}{2}(\nabla \cdot(\rho \mathbf{u}) \mathbf{u}, \mathbf{u})+(\boldsymbol{J} \cdot \nabla \mathbf{u}, \mathbf{u})+\frac{1}{2}(\nabla \cdot \boldsymbol{J} \mathbf{u}, \mathbf{u})
\end{aligned}
$$

where $\boldsymbol{J}, \rho, \nu$ are given in $(2.7)-(2.9)$, and $H(\phi)=\frac{f(\phi)-s \phi}{\sqrt{\int_{\Omega}\left(F(\phi)-\frac{s}{2} \phi^{2}\right) \mathrm{d} \boldsymbol{x}+B}}$.

Since the variables $U, Q, R$ are ODEs for time, the boundary conditions of the new system (3.4)-(3.10) are still (2.11), and the initial conditions read as

$$
\left\{\begin{array}{l}
\left.\mathbf{u}\right|_{(t=0)}=\mathbf{u}^{0},\left.p\right|_{(t=0)}=p^{0},\left.\phi\right|_{(t=0)}=\phi^{0} \\
\left.U\right|_{(t=0)}=\sqrt{\int_{\Omega}\left(F\left(\phi^{0}\right)-\frac{s}{2}\left(\phi^{0}\right)^{2}\right) \mathrm{d} \boldsymbol{x}+B},\left.Q\right|_{(t=0)}=1,\left.R\right|_{(t=0)}=1 .
\end{array}\right.
$$


Note that there exist some modifications appear in the system (3.4)-(3.10). Here we highlight that even after these modifications, the new system is still equivalent to the original system (2.2)-(2.5). For the momentum equation (3.6), an extra term, $\frac{1}{2} \rho_{t} \mathbf{u}+R\left(\frac{1}{2} \nabla \cdot(\rho \mathbf{u}) \mathbf{u}+\frac{1}{2} \nabla \cdot \boldsymbol{J} \mathbf{u}\right)$, are added. This extra term is actually zero from (2.18) and $R=1$. We also multiply some terms, e.g., $\phi \nabla \mu$ and $R \rho(\mathbf{u} \cdot \nabla) \mathbf{u}$ by $Q$ or $R$. Since $Q=R=1$, these modifications will not change the system.

The new system (3.4)-(3.10) also follows the law of energy dissipation, which is derived in the following theorem.

Theorem 3.1. The new system (3.4)-(3.10) holds

$$
\frac{\mathrm{d}}{\mathrm{d} t} E(\rho, \mathbf{u}, \phi, U, Q, R)=-M\|\nabla \mu\|^{2}-\frac{1}{2} \int_{\Omega} \nu|D(\mathbf{u})|^{2} \mathrm{~d} \boldsymbol{x} \leq 0,
$$

where

$$
E(\rho, \mathbf{u}, \phi, U, Q, R)=\left(\int_{\Omega}\left(\frac{1}{2} \rho|\mathbf{u}|^{2}+\lambda \frac{\epsilon}{2}|\nabla \phi|^{2}+\lambda \frac{s}{2 \epsilon}|\phi|^{2}\right) \mathrm{d} \boldsymbol{x}+\frac{\lambda}{\epsilon}|U|^{2}+\frac{|Q|^{2}}{2}+\frac{|R|^{2}}{2}-B-1\right) .
$$

Proof. By multiplying the $L^{2}$ inner product of (3.4) with $\mu$, and of (3.5) with $-\phi_{t}$, we get

$$
\begin{aligned}
\left(\phi_{t}, \mu\right) & =-\underbrace{Q(\nabla \cdot(\mathbf{u} \phi), \mu)}_{\mathrm{I}}-M\|\nabla \mu\|^{2}, \\
-\left(\mu, \phi_{t}\right)+\lambda \frac{\mathrm{d}}{\mathrm{d} t}\left(\int_{\Omega}\left(\frac{\epsilon}{2}|\nabla \phi|^{2}+\frac{s}{2 \epsilon}|\phi|^{2}\right) \mathrm{d} \boldsymbol{x}\right) & =-\frac{\lambda}{\epsilon} U\left(H, \phi_{t}\right) .
\end{aligned}
$$

By taking the $L^{2}$ inner product of (3.6) with $\mathbf{u}$ and using integration by parts and the divergence-free condition (3.7), we obtain

$$
\begin{aligned}
\frac{\mathrm{d}}{\mathrm{d} t} \int_{\Omega} \frac{1}{2} \rho|\mathbf{u}|^{2} \mathrm{~d} \boldsymbol{x}= & -\frac{1}{2} \int_{\Omega} \nu|D(\mathbf{u})|^{2} \mathrm{~d} \boldsymbol{x}-\underbrace{Q(\phi \nabla \mu, \mathbf{u})}_{\text {II }} \\
& -\underbrace{R(\rho(\mathbf{u} \cdot \nabla) \mathbf{u}, \mathbf{u})}_{\text {III }}-\underbrace{\frac{1}{2} R(\nabla \cdot(\rho \mathbf{u}) \mathbf{u}, \mathbf{u})}_{\text {IV }}-\underbrace{R(\boldsymbol{J} \cdot \nabla \mathbf{u}, \mathbf{u})}_{\mathrm{V}}-\underbrace{\frac{1}{2} R(\nabla \cdot \boldsymbol{J} \mathbf{u}, \mathbf{u})}_{\text {VI }} .
\end{aligned}
$$

By multiplying (3.8) with $\frac{2 \lambda}{\epsilon} U,(3.9)$ with $Q$, and (3.10) with $R$, we obtain

$$
\begin{aligned}
\frac{\mathrm{d}}{\mathrm{d} t}\left(\frac{\lambda}{\epsilon}|U|^{2}\right) & =\frac{\lambda}{\epsilon} U\left(H, \phi_{t}\right), \\
\frac{\mathrm{d}}{\mathrm{d} t}\left(\frac{|Q|^{2}}{2}\right) & =\underbrace{Q(\nabla \cdot(\mathbf{u} \phi), \mu)}_{\mathrm{I}_{1}}+\underbrace{Q(\phi \nabla \mu, \mathbf{u})}_{\mathrm{II}_{1}}, \\
\frac{\mathrm{d}}{\mathrm{d} t}\left(\frac{|R|^{2}}{2}\right) & =\underbrace{R(\rho(\mathbf{u} \cdot \nabla) \mathbf{u}, \mathbf{u})}_{\mathrm{III}_{1}}+\underbrace{\frac{1}{2} R(\nabla \cdot(\rho \mathbf{u}) \mathbf{u}, \mathbf{u})}_{\mathrm{IV}_{1}}+\underbrace{R(\boldsymbol{J} \cdot \nabla \mathbf{u}, \mathbf{u})}_{\mathrm{V}_{1}}+\underbrace{\frac{1}{2} R(\nabla \cdot \boldsymbol{J} \mathbf{u}, \mathbf{u})}_{\mathrm{VI}_{1}} .
\end{aligned}
$$

By combining the above six equations (3.14)-(3.19) and noting that the two terms marked with the same Roman numerals cancel each other out, we obtain the law of energy dissipation (3.12).

Remark 3.1. Note that we add the constant $-B-1$ in the above modified energy (3.13) to make it consistent with the original energy (2.13) for the continuous case. This is because in the continuous case, we have (3.1) and $Q(t)=R(t)=1$. Therefore, after adding the constant $-B-1$ in (3.13), this modified energy just becomes (2.13). 


\subsection{Numerical scheme}

In this subsection, we develop a fully-discrete numerical scheme to solve the system (3.4)-(3.10) which is an equivalent system of (2.2)-(2.5). We denote $\delta t>0$ as a time step size and $t^{n}=n \delta t$ for $0 \leq n \leq N$ with $T=N \delta t$. Let $\psi^{n}$ be the numerical approximation to the function $\left.\psi(\cdot, t)\right|_{t=t^{n}}$.

Some finite dimensional discrete subspaces are introduced as follows. Assuming that the polygonal/polyhedral domain $\Omega$ is discretized by a conforming and shape regular triangulation/tetrahedron mesh $\mathcal{T}_{h}$ that is composed by open disjoint elements $K$ such that $\bar{\Omega}=\bigcup_{K \in \mathcal{T}_{h}} \bar{K}$. We use $\mathcal{P}_{l}$ to denote the space of polynomials of total degree at most $l$ and define the following finite element spaces:

$$
\begin{aligned}
Y_{h} & =\left\{X \in C^{0}(\Omega):\left.X\right|_{K} \in \mathcal{P}_{l_{1}}(K), \forall K \in \mathcal{T}_{h}\right\}, \\
\boldsymbol{V}_{h} & =\left\{\boldsymbol{v} \in C^{0}(\Omega)^{d}:\left.\boldsymbol{v}\right|_{K} \in \mathcal{P}_{l_{2}}(K)^{d}, \forall K \in \mathcal{T}_{h}\right\} \cap H_{0}^{1}(\Omega)^{d}, \\
\boldsymbol{U}_{h} & =\left\{\boldsymbol{v} \in C^{0}(\Omega)^{d}:\left.\boldsymbol{v}\right|_{K} \in \mathcal{P}_{l_{2}}(K)^{d}, \forall K \in \mathcal{T}_{h}\right\} \cap H^{1}(\Omega)^{d}, \\
O_{h} & =\left\{q \in C^{0}(\Omega):\left.q\right|_{K} \in \mathcal{P}_{l_{2}-1}(K), \forall K \in \mathcal{T}_{h}\right\} \cap L_{0}^{2}(\Omega),
\end{aligned}
$$

where $H_{0}^{1}(\Omega)=\left\{u \in H^{1}(\Omega):\left.u\right|_{\partial \Omega}=0\right\}$ and $L_{0}^{2}(\Omega)=\left\{q \in L^{2}(\Omega): \int_{\Omega} q \mathrm{~d} x=0\right\}$. Hence,

$$
Y_{h} \subset H^{1}(\Omega), \boldsymbol{V}_{h} \subset H_{0}^{1}(\Omega)^{d}, O_{h} \subset L_{0}^{2}(\Omega) .
$$

Besides, we assume the pair of spaces $\left(\boldsymbol{V}_{h}, O_{h}\right)$ satisfy the inf-sup condition [14]:

$$
\beta\|q\| \leq \sup _{\boldsymbol{v} \in \boldsymbol{V}_{h}} \frac{(\nabla \cdot \boldsymbol{v}, q)}{\|\nabla \boldsymbol{v}\|}, \quad \forall q \in O_{h},
$$

where the constant $\beta$ only depends on $\Omega$. A well known inf-sup stable pair $\left(\boldsymbol{V}_{h}, O_{h}\right)$ is the Taylor-Hood element $[14]$.

The semi-discrete formulations of the system (3.4)-(3.7) in the weak form reads as: find $(\phi, \mu, \mathbf{u}, p) \in$ $Y_{h} \times Y_{h} \times \boldsymbol{V}_{h} \times O_{h}$, such that

$$
\begin{aligned}
\left(\phi_{t}, w\right)-Q(\mathbf{u} \phi, \nabla w)= & -M(\nabla \mu, \nabla w) \\
(\mu, \psi)= & \lambda \epsilon(\nabla \phi, \nabla \psi)+\lambda \frac{s}{\epsilon}(\phi, \psi)+\lambda \frac{1}{\epsilon} U(H, \psi), \\
\left(\rho \mathbf{u}_{t}, \boldsymbol{v}\right)+ & \frac{1}{2}\left(\rho_{t} \mathbf{u}, \boldsymbol{v}\right)+\frac{1}{2}(\nu D(\mathbf{u}), D(\boldsymbol{v}))+(\nabla p, \boldsymbol{v})+Q(\phi \nabla \mu, \boldsymbol{v}) \\
& +R(\rho(\mathbf{u} \cdot \nabla) \mathbf{u}, \boldsymbol{v})+\frac{1}{2} R(\nabla \cdot(\rho \mathbf{u}) \mathbf{u}, \boldsymbol{v})+R(\boldsymbol{J} \cdot \nabla \mathbf{u}, \boldsymbol{v})+\frac{1}{2} R(\nabla \cdot \boldsymbol{J} \mathbf{u}, \boldsymbol{v})=0, \\
(\nabla \cdot \mathbf{u}, q)= & 0 \\
U_{t}= & \frac{1}{2}\left(H, \phi_{t}\right), \\
Q_{t}= & -(\phi \mathbf{u}, \nabla \mu)+(\phi \nabla \mu, \mathbf{u}), \\
R_{t}= & (\rho(\mathbf{u} \cdot \nabla) \mathbf{u}, \mathbf{u})+\frac{1}{2}(\nabla \cdot(\rho \mathbf{u}) \mathbf{u}, \mathbf{u})+(\boldsymbol{J} \cdot \nabla \mathbf{u}, \mathbf{u})+\frac{1}{2}(\nabla \cdot \boldsymbol{J} \mathbf{u}, \mathbf{u}),
\end{aligned}
$$

for $(\psi, w, \boldsymbol{v}, q) \in Y_{h} \times Y_{h} \times \boldsymbol{V}_{h} \times O_{h}$, where $\rho, \nu, \boldsymbol{J}$ is given in (2.7)-(2.9).

By combining the penalty method for the Navier-Stokes equation, the Strang operator splitting method, we construct a fully-discrete scheme as follows: given $\mathbf{u}_{h}^{n}, p_{h}^{n}, \phi_{h}^{n}, U^{n}, Q^{n}, R^{n}$,

Step 1. Find $\phi_{h}^{n+1} \in Y_{h}, \mu_{h}^{n+1} \in Y_{h}, \tilde{\mathbf{u}}_{h}^{n+1} \in \boldsymbol{U}_{h}, U^{n+1}, Q^{n+1} \in \mathscr{R}$ such that

$$
\left(\frac{\phi_{h}^{n+1}-\phi_{h}^{n}}{\delta t}, w_{h}\right)-Q^{n+1}\left(\mathbf{u}_{h}^{n} \phi_{h}^{n}, \nabla w_{h}\right)=-M\left(\nabla \mu_{h}^{n+1}, \nabla w_{h}\right),
$$




$$
\begin{aligned}
& \left(\mu_{h}^{n+1}, \psi_{h}\right)=\lambda \epsilon\left(\nabla \phi_{h}^{n+1}, \nabla \psi_{h}\right)+\lambda \frac{s}{\epsilon}\left(\phi_{h}^{n+1}, \psi_{h}\right)+\lambda \frac{1}{\epsilon} U^{n+1}\left(H_{h}^{n}, \psi_{h}\right), \\
& U^{n+1}-U^{n}=\frac{1}{2}\left(H_{h}^{n}, \phi_{h}^{n+1}-\phi_{h}^{n}\right), \\
& \left(\rho_{h}^{n} \frac{\tilde{\mathbf{u}}_{h}^{n+1}-\mathbf{u}_{h}^{n}}{\delta t}, \boldsymbol{v}_{h}\right)+Q^{n+1}\left(\phi_{h}^{n} \nabla \mu_{h}^{n}, \boldsymbol{v}_{h}\right)=0, \\
& \frac{Q^{n+1}-Q^{n}}{\delta t}=-\left(\mathbf{u}_{h}^{n} \phi_{h}^{n}, \nabla \mu_{h}^{n+1}\right)+\left(\phi_{h}^{n} \nabla \mu_{h}^{n}, \tilde{\mathbf{u}}_{h}^{n+1}\right) .
\end{aligned}
$$

Step 2. Find $\mathbf{u}_{h}^{n+1} \in \boldsymbol{V}_{h}$, and $R^{n+1} \in \mathscr{R}$ such that

$$
\begin{aligned}
\left(\rho_{h}^{n} \frac{\mathbf{u}_{h}^{n+1}-\tilde{\mathbf{u}}_{h}^{n+1}}{\delta t}, \boldsymbol{v}_{h}\right)+ & \frac{1}{2}\left(\frac{\rho_{h}^{n+1}-\rho_{h}^{n}}{\delta t} \mathbf{u}_{h}^{n+1}, \boldsymbol{v}_{h}\right)+\frac{1}{2}\left(\nu_{h}^{n+1} D\left(\mathbf{u}_{h}^{n+1}\right), D\left(\boldsymbol{v}_{h}\right)\right) \\
+ & \left(\nabla\left(2 p_{h}^{n}-p_{h}^{n-1}\right), \boldsymbol{v}_{h}\right) \\
+ & R^{n+1}\left(\rho_{h}^{n}\left(\mathbf{u}_{h}^{n} \cdot \nabla\right) \mathbf{u}_{h}^{n}, \boldsymbol{v}_{h}\right)+\frac{1}{2} R^{n+1}\left(\nabla \cdot\left(\rho_{h}^{n} \mathbf{u}_{h}^{n}\right) \mathbf{u}_{h}^{n}, \boldsymbol{v}_{h}\right) \\
+ & R^{n+1}\left(\boldsymbol{J}_{h}^{n} \cdot \nabla \mathbf{u}_{h}^{n}, \boldsymbol{v}_{h}\right)+\frac{1}{2} R^{n+1}\left(\nabla \cdot \boldsymbol{J}_{h}^{n} \mathbf{u}_{h}^{n}, \boldsymbol{v}_{h}\right)=0, \\
\frac{R^{n+1}-R^{n}}{\delta t}= & \left(\rho_{h}^{n}\left(\mathbf{u}_{h}^{n} \cdot \nabla\right) \mathbf{u}_{h}^{n}, \mathbf{u}_{h}^{n+1}\right)+\frac{1}{2}\left(\nabla \cdot\left(\rho_{h}^{n} \mathbf{u}_{h}^{n}\right) \mathbf{u}_{h}^{n}, \mathbf{u}_{h}^{n+1}\right) \\
& +\left(\boldsymbol{J}_{h}^{n} \cdot \nabla \mathbf{u}_{h}^{n}, \mathbf{u}_{h}^{n+1}\right)+\frac{1}{2}\left(\nabla \cdot \boldsymbol{J}_{h}^{n} \mathbf{u}_{h}^{n}, \mathbf{u}_{h}^{n+1}\right) .
\end{aligned}
$$

Step 3. Find $p_{h}^{n+1} \in O_{h}$ such that

$$
\left(\nabla\left(p_{h}^{n+1}-p_{h}^{n}\right), \nabla q_{h}\right)=\frac{\chi}{\delta t}\left(\mathbf{u}_{h}^{n+1}, \nabla q_{h}\right),
$$

for any $\psi_{h} \in Y_{h}, w_{h} \in Y_{h}, \boldsymbol{v}_{h} \in \boldsymbol{V}_{h}, q_{h} \in O_{h}$, where

$$
\left\{\begin{array}{l}
H_{h}^{n}=H\left(\phi_{h}^{n}\right), \boldsymbol{J}_{h}^{n}=\boldsymbol{J}\left(\mu_{h}^{n}\right), \chi=\frac{1}{2} \min \left(\rho_{1}, \rho_{2}\right), \\
\hat{\phi}= \begin{cases}\phi, & |\phi|<1 \\
\operatorname{sign}(\phi), & |\phi|>1,\end{cases} \\
\rho_{h}^{n+1}=\frac{\rho_{1}-\rho_{2}}{2} \hat{\phi}_{h}^{n+1}+\frac{\rho_{1}+\rho_{2}}{2}, \nu_{h}^{n+1}=\frac{\nu_{1}-\nu_{2}}{2} \hat{\phi}_{h}^{n+1}+\frac{\nu_{1}+\nu_{2}}{2} .
\end{array}\right.
$$

Some explanations of the scheme (3.29)-(3.36) are given through the following remarks.

Remark 3.2. We adopt the penalty method given in $[19,25,33]$ to discretize the Navier-Stokes equations with variable density. In this way, the computation of pressure is not only completely decoupled from the momentum equation, but also avoids solving the elliptic equation with $1 / \rho$ as the variable coefficient, which can greatly reduce the calculation cost and increase the computational efficiency. Note that we choose $p_{h}^{-1}=p_{h}^{0}$ in practice.

Remark 3.3. Note that we use the implicit and explicit combination method to handle nonlinear terms. For instance, the term $Q(\mathbf{u} \phi, \nabla w)$ in (3.22) is discretized as $Q^{n+1}\left(\mathbf{u}_{h}^{n} \phi_{h}^{n}, \nabla w_{h}\right)$ in (3.29). Thus the scheme is linear.

Remark 3.4. We use the cut-off function to update the density $\rho^{n+1}$ in (3.37), which gives

$$
\rho_{h}^{n+1} \geq \min \left(\rho_{1}, \rho_{2}\right), \quad \nu_{h}^{n+1} \geq \min \left(\nu_{1}, \nu_{2}\right),
$$

which is quite important to derive the energy stability, see the proof in Theorem 3.2. In fact, the cut-off technique of $\phi$ is a commonly-used technique for the variable density phase-field model, either for the modeling, or the numerical algorithm, see also in $[13,27,32,33,44]$. 
Remark 3.5. In step 1 , to obtain the intermediate velocity $\tilde{\mathbf{u}}_{h}^{n+1}$, we split the surface tension term $Q^{n+1} \phi_{h}^{n} \nabla \mu_{h}^{n}$ from the momentum equation, which is the so-called first-order operator Strang-splitting method, see also in $[4,25,33]$. However, there are some slight differences in the discretization of this specific term between our developed scheme and the scheme developed in $[4,25,33]$. We refer to the detailed comparisons given in Remark 3.7.

The following theorem shows the unconditional energy stability held by the scheme (3.29)-(3.36).

Theorem 3.2. The solutions of the time-discrete scheme (3.29)-(3.36) satisfies

$$
E_{h}^{n+1} \leq E_{h}^{n}-\frac{1}{2} \delta t\left\|\sqrt{\nu_{h}^{n+1}} D\left(\mathbf{u}_{h}^{n+1}\right)\right\|^{2}-M \delta t\left\|\nabla \mu_{h}^{n+1}\right\|^{2},
$$

where

$$
\begin{aligned}
E_{h}^{n+1}= & \frac{1}{2}\left\|\sigma_{h}^{n+1} \mathbf{u}_{h}^{n+1}\right\|^{2}+\frac{\delta t^{2}}{2 \chi}\left\|\nabla p_{h}^{n+1}\right\|^{2}+\lambda \frac{\epsilon}{2}\left\|\nabla \phi_{h}^{n+1}\right\|^{2}+\lambda \frac{s}{2 \epsilon}\left\|\phi_{h}^{n+1}\right\|^{2} \\
& +\frac{\lambda}{\epsilon}\left|U^{n+1}\right|^{2}+\frac{1}{2}\left|Q^{n+1}\right|^{2}+\frac{1}{2}\left|R^{n+1}\right|^{2}-B-1,
\end{aligned}
$$

and $\sigma_{h}^{n+1}=\sqrt{\rho_{h}^{n+1}}$.

Proof. By taking $\boldsymbol{v}_{h}=2 \delta t \mathbf{u}_{h}^{n+1}$ in (3.34) and using the following identity

$$
2(a-b) a=a^{2}-b^{2}+(a-b)^{2},
$$

we obtain

$$
\begin{aligned}
\left\|\sigma_{h}^{n+1} \mathbf{u}_{h}^{n+1}\right\|^{2}- & \left\|\sigma_{h}^{n} \tilde{\mathbf{u}}_{h}^{n+1}\right\|^{2}+\left\|\sigma_{h}^{n}\left(\mathbf{u}_{h}^{n+1}-\tilde{\mathbf{u}}_{h}^{n+1}\right)\right\|^{2}+\delta t\left\|\sqrt{\nu_{h}^{n+1}} D\left(\mathbf{u}_{h}^{n+1}\right)\right\|^{2} \\
- & 2 \delta t\left(\nabla\left(p_{h}^{n+1}-2 p_{h}^{n}+p_{h}^{n-1}\right), \mathbf{u}_{h}^{n+1}\right)+2 \delta t\left(\nabla p_{h}^{n+1}, \mathbf{u}_{h}^{n+1}\right) \\
= & -2 \delta t R^{n+1}\left(\left(\rho_{h}^{n}\left(\mathbf{u}_{h}^{n} \cdot \nabla\right) \mathbf{u}_{h}^{n}, \mathbf{u}_{h}^{n+1}\right)+\frac{1}{2}\left(\nabla \cdot\left(\rho_{h}^{n} \mathbf{u}_{h}^{n}\right) \mathbf{u}_{h}^{n}, \mathbf{u}_{h}^{n+1}\right)\right) \\
& -2 \delta t R^{n+1}\left(\left(\boldsymbol{J}_{h}^{n} \cdot \nabla \mathbf{u}_{h}^{n}, \mathbf{u}_{h}^{n+1}\right)+\frac{1}{2}\left(\nabla \cdot \boldsymbol{J}_{h}^{n} \mathbf{u}_{h}^{n}, \mathbf{u}_{h}^{n+1}\right)\right) .
\end{aligned}
$$

By taking $\boldsymbol{v}_{h}=2 \delta t \tilde{\mathbf{u}}^{n+1}$ in (3.32), we obtain

$$
\left\|\sigma_{h}^{n} \tilde{\mathbf{u}}_{h}^{n+1}\right\|^{2}-\left\|\sigma_{h}^{n} \mathbf{u}_{h}^{n}\right\|^{2}+\left\|\sigma_{h}^{n}\left(\tilde{\mathbf{u}}_{h}^{n+1}-\mathbf{u}_{h}^{n}\right)\right\|^{2}=-2 \delta t Q^{n+1}\left(\phi_{h}^{n} \nabla \mu_{h}^{n}, \tilde{\mathbf{u}}_{h}^{n+1}\right) .
$$

By combining (3.42) and (3.43), we obtain

$$
\begin{aligned}
\left\|\sigma_{h}^{n+1} \mathbf{u}_{h}^{n+1}\right\|^{2}- & \left\|\sigma_{h}^{n} \mathbf{u}_{h}^{n}\right\|^{2}+\left\|\sigma_{h}^{n}\left(\mathbf{u}_{h}^{n+1}-\tilde{\mathbf{u}}_{h}^{n+1}\right)\right\|^{2}+\left\|\sigma_{h}^{n}\left(\tilde{\mathbf{u}}_{h}^{n+1}-\mathbf{u}_{h}^{n}\right)\right\|^{2} \\
+ & \delta t\left\|\sqrt{\nu_{h}^{n+1}} D\left(\mathbf{u}_{h}^{n+1}\right)\right\|^{2}-2 \delta t\left(\nabla\left(p_{h}^{n+1}-2 p_{h}^{n}+p_{h}^{n-1}\right), \mathbf{u}_{h}^{n+1}\right)+2 \delta t\left(\nabla p_{h}^{n+1}, \mathbf{u}_{h}^{n+1}\right) \\
= & -2 \delta t R^{n+1}\left(\left(\rho_{h}^{n}\left(\mathbf{u}_{h}^{n} \cdot \nabla\right) \mathbf{u}_{h}^{n}, \mathbf{u}_{h}^{n+1}\right)+\frac{1}{2}\left(\nabla \cdot\left(\rho_{h}^{n} \mathbf{u}_{h}^{n}\right) \mathbf{u}_{h}^{n}, \mathbf{u}_{h}^{n+1}\right)\right) \\
& -2 \delta t R^{n+1}\left(\left(\boldsymbol{J}_{h}^{n} \cdot \nabla \mathbf{u}_{h}^{n}, \mathbf{u}_{h}^{n+1}\right)+\frac{1}{2}\left(\nabla \cdot \boldsymbol{J}_{h}^{n} \mathbf{u}_{h}^{n}, \mathbf{u}_{h}^{n+1}\right)\right)-2 \delta t Q^{n+1}\left(\phi_{h}^{n} \nabla \mu_{h}^{n}, \tilde{\mathbf{u}}_{h}^{n+1}\right) .
\end{aligned}
$$

We take $q_{h}=-\frac{2 \delta t^{2}}{\chi}\left(p_{h}^{n+1}-2 p_{h}^{n}+p_{h}^{n-1}\right)$ in (3.36) to obtain

$$
\begin{aligned}
-2 \delta t\left(\nabla\left(p_{h}^{n+1}-2 p_{h}^{n}+p_{h}^{n-1}\right), \mathbf{u}_{h}^{n+1}\right)= & -\frac{\delta t^{2}}{\chi}\left(\left\|\nabla p_{h}^{n+1}-\nabla p_{h}^{n}\right\|^{2}-\left\|\nabla p_{h}^{n}-\nabla p_{h}^{n-1}\right\|^{2}\right. \\
& \left.+\left\|\nabla p_{h}^{n+1}-2 \nabla p_{h}^{n}+\nabla p_{h}^{n-1}\right\|^{2}\right) .
\end{aligned}
$$


We take $q_{h}=\frac{2 \delta t^{2}}{\chi} p_{h}^{n+1}$ in (3.36) to obtain

$$
2 \delta t\left(\nabla p_{h}^{n+1}, \mathbf{u}_{h}^{n+1}\right)=\frac{\delta t^{2}}{\chi}\left(\left\|\nabla p_{h}^{n+1}\right\|^{2}-\left\|\nabla p_{h}^{n}\right\|^{2}+\left\|\nabla p_{h}^{n+1}-\nabla p_{h}^{n}\right\|^{2}\right) .
$$

By combining (3.45) and (3.46), we derive

$$
\begin{aligned}
-2 \delta t\left(\nabla\left(p_{h}^{n+1}-2 p_{h}^{n}+p_{h}^{n-1}\right), \mathbf{u}_{h}^{n+1}\right)+2 \delta t\left(\nabla p_{h}^{n+1}, \mathbf{u}_{h}^{n+1}\right)= & \frac{\delta t^{2}}{\chi}\left(\left\|\nabla p_{h}^{n+1}\right\|^{2}-\left\|\nabla p_{h}^{n}\right\|^{2}\right)+\frac{\delta t^{2}}{\chi}\left\|\nabla p_{h}^{n}-\nabla p_{h}^{n-1}\right\|^{2} \\
& -\frac{\delta t^{2}}{\chi}\left\|\nabla p_{h}^{n+1}-2 \nabla p_{h}^{n}+\nabla p_{h}^{n-1}\right\|^{2}
\end{aligned}
$$

where we use the following identity:

$$
2(a-b)(a-2 b+c)=(a-b)^{2}-(b-c)^{2}+(a-2 b+c)^{2} .
$$

We subtract (3.36) at $(n+1)$-step and $n$-step to obtain

$$
\left(\nabla\left(p_{h}^{n+1}-2 p_{h}^{n}+p_{h}^{n-1}\right), \nabla q_{h}\right)=\frac{\chi}{\delta t}\left(\mathbf{u}_{h}^{n+1}-\mathbf{u}_{h}^{n}, \nabla q_{h}\right) .
$$

By taking $q_{h}=p_{h}^{n+1}-2 p_{h}^{n}+p_{h}^{n-1}$ in (3.49), we derive

$$
\begin{aligned}
\left\|\nabla\left(p_{h}^{n+1}-2 p_{h}^{n}+p_{h}^{n-1}\right)\right\|^{2} & =\frac{\chi}{\delta t}\left(\mathbf{u}_{h}^{n+1}-\mathbf{u}_{h}^{n}, \nabla\left(p_{h}^{n+1}-2 p_{h}^{n}+p_{h}^{n-1}\right)\right) \\
& \leq \frac{\chi^{2}}{2 \delta t^{2}}\left\|\mathbf{u}_{h}^{n+1}-\mathbf{u}_{h}^{n}\right\|^{2}+\frac{1}{2}\left\|\nabla\left(p_{h}^{n+1}-2 p_{h}^{n}+p_{h}^{n-1}\right)\right\|^{2},
\end{aligned}
$$

where we use the Cauchy-Schwarz inequality. Hence (3.50) implies

$$
\left\|\nabla\left(p_{h}^{n+1}-2 p_{h}^{n}+p_{h}^{n-1}\right)\right\|^{2} \leq \frac{\chi^{2}}{\delta t^{2}}\left\|\mathbf{u}_{h}^{n+1}-\mathbf{u}_{h}^{n}\right\|^{2} .
$$

By multiplying (3.51) with $\frac{\delta t^{2}}{\chi}$ and applying $\chi=\frac{1}{2} \min \left(\rho_{1}, \rho_{2}\right) \leq \frac{1}{2} \rho_{h}^{n}$, we derive

$$
\frac{\delta t^{2}}{\chi}\left\|\nabla\left(p_{h}^{n+1}-2 p_{h}^{n}+p_{h}^{n-1}\right)\right\|^{2} \leq \chi\left\|\mathbf{u}_{h}^{n+1}-\mathbf{u}_{h}^{n}\right\|^{2} \leq \frac{1}{2}\left\|\sigma_{h}^{n}\left(\mathbf{u}_{h}^{n+1}-\mathbf{u}_{h}^{n}\right)\right\|^{2} .
$$

Using the inequality $\frac{1}{2}(a+b)^{2} \leq a^{2}+b^{2}$, we obtain

$$
\frac{1}{2}\left\|\sigma_{h}^{n}\left(\mathbf{u}_{h}^{n+1}-\mathbf{u}_{h}^{n}\right)\right\|^{2} \leq\left\|\sigma_{h}^{n}\left(\mathbf{u}_{h}^{n+1}-\tilde{\mathbf{u}}_{h}^{n+1}\right)\right\|^{2}+\left\|\sigma_{h}^{n}\left(\tilde{\mathbf{u}}_{h}^{n+1}-\mathbf{u}_{h}^{n}\right)\right\|^{2} .
$$

Therefore, by combining (3.47), (3.52) and (3.53), we obtain

$$
\begin{aligned}
-2 \delta t\left(\nabla\left(p_{h}^{n+1}-2 p_{h}^{n}+p_{h}^{n-1}\right), \mathbf{u}_{h}^{n+1}\right)+2 \delta t\left(\nabla p_{h}^{n+1}, \mathbf{u}_{h}^{n+1}\right) \geq & \frac{\delta t^{2}}{\chi}\left(\left\|\nabla p_{h}^{n+1}\right\|^{2}-\left\|\nabla p_{h}^{n}\right\|^{2}\right)+\frac{\delta t^{2}}{\chi}\left\|\nabla p_{h}^{n}-\nabla p_{h}^{n-1}\right\|^{2} \\
& -\left\|\sigma_{h}^{n}\left(\mathbf{u}_{h}^{n+1}-\tilde{\mathbf{u}}_{h}^{n+1}\right)\right\|^{2}-\left\|\sigma_{h}^{n}\left(\tilde{\mathbf{u}}_{h}^{n+1}-\mathbf{u}_{h}^{n}\right)\right\|^{2}
\end{aligned}
$$


Hence, from (3.42) and (3.54), we derive

$$
\begin{aligned}
\left\|\sigma_{h}^{n+1} \mathbf{u}_{h}^{n+1}\right\|^{2}- & \left\|\sigma_{h}^{n} \mathbf{u}_{h}^{n}\right\|^{2}+\delta t\left\|\sqrt{\nu_{h}^{n+1}} D\left(\mathbf{u}_{h}^{n+1}\right)\right\|^{2} \\
+ & \frac{\delta t^{2}}{\chi}\left(\left\|\nabla p_{h}^{n+1}\right\|^{2}-\left\|\nabla p_{h}^{n}\right\|^{2}\right)+\frac{\delta t^{2}}{\chi}\left\|\nabla p_{h}^{n}-\nabla p_{h}^{n-1}\right\|^{2} \\
\leq & -2 \delta t R^{n+1}\left(\left(\rho_{h}^{n}\left(\mathbf{u}_{h}^{n} \cdot \nabla\right) \mathbf{u}_{h}^{n}, \mathbf{u}_{h}^{n+1}\right)+\frac{1}{2}\left(\nabla \cdot\left(\rho_{h}^{n} \mathbf{u}_{h}^{n}\right) \mathbf{u}_{h}^{n}, \mathbf{u}_{h}^{n+1}\right)\right) \\
& -2 \delta t R^{n+1}\left(\left(\boldsymbol{J}_{h}^{n} \cdot \nabla \mathbf{u}_{h}^{n}, \mathbf{u}_{h}^{n+1}\right)+\frac{1}{2}\left(\nabla \cdot \boldsymbol{J}_{h}^{n} \mathbf{u}_{h}^{n}, \mathbf{u}_{h}^{n+1}\right)\right) \\
& -2 \delta t Q^{n+1}\left(\phi_{h}^{n} \nabla \mu_{h}^{n}, \tilde{\mathbf{u}}_{h}^{n+1}\right) .
\end{aligned}
$$

We take $w_{h}=2 \delta t \mu_{h}^{n+1}$ in (3.29) to get

$$
2\left(\phi_{h}^{n+1}-\phi_{h}^{n}, \mu_{h}^{n+1}\right)+2 M \delta t\left\|\nabla \mu_{h}^{n+1}\right\|^{2}=2 \delta t Q^{n+1}\left(\mathbf{u}_{h}^{n} \phi_{h}^{n}, \nabla \mu_{h}^{n+1}\right) .
$$

We take $\psi_{h}=-2\left(\phi_{h}^{n+1}-\phi_{h}^{n}\right)$ in (3.30) to get

$$
\begin{aligned}
-2\left(\phi_{h}^{n+1}-\phi_{h}^{n}, \mu_{h}^{n+1}\right) & +\lambda \epsilon\left(\left\|\nabla \phi_{h}^{n+1}\right\|^{2}-\left\|\nabla \phi_{h}^{n}\right\|^{2}+\left\|\nabla \phi_{h}^{n+1}-\nabla \phi_{h}^{n}\right\|^{2}\right) \\
& +\lambda \frac{s}{\epsilon}\left(\left\|\phi_{h}^{n+1}\right\|^{2}-\left\|\phi_{h}^{n}\right\|^{2}+\left\|\phi_{h}^{n+1}-\phi_{h}^{n}\right\|^{2}\right)=-\frac{2 \lambda}{\epsilon} U^{n+1}\left(H_{h}^{n}, \phi_{h}^{n+1}-\phi_{h}^{n}\right) .
\end{aligned}
$$

We multiply (3.31) with $\frac{4 \lambda}{\epsilon} U^{n+1}$ to get

$$
\frac{2 \lambda}{\epsilon}\left(\left|U^{n+1}\right|^{2}-\left|U^{n}\right|^{2}+\left|U^{n+1}-U^{n}\right|^{2}\right)=\frac{2 \lambda}{\epsilon} U^{n+1}\left(H_{h}^{n}, \phi_{h}^{n+1}-\phi_{h}^{n}\right) .
$$

We multiply (3.33) with $2 \delta t Q^{n+1}$ to get

$$
\left(\left|Q^{n+1}\right|^{2}-\left|Q^{n}\right|^{2}+\left|Q^{n+1}-Q^{n}\right|^{2}\right)=-2 \delta t Q^{n+1}\left(\mathbf{u}_{h}^{n} \phi_{h}^{n}, \nabla \mu_{h}^{n+1}\right)+2 \delta t Q^{n+1}\left(\phi_{h}^{n} \nabla \mu_{h}^{n}, \tilde{\mathbf{u}}_{h}^{n+1}\right) .
$$

We multiply (3.35) with $2 \delta t R^{n+1}$ to get

$$
\begin{aligned}
\left(\left|R^{n+1}\right|^{2}-\left|R^{n}\right|^{2}+\left|R^{n+1}-R^{n}\right|^{2}\right)= & 2 \delta t R^{n+1}\left(\left(\rho_{h}^{n}\left(\mathbf{u}_{h}^{n} \cdot \nabla\right) \mathbf{u}_{h}^{n}, \mathbf{u}_{h}^{n+1}\right)+\frac{1}{2}\left(\nabla \cdot\left(\rho_{h}^{n} \mathbf{u}_{h}^{n}\right) \mathbf{u}_{h}^{n}, \mathbf{u}_{h}^{n+1}\right)\right) \\
& +2 \delta t R^{n+1}\left(\left(\boldsymbol{J}_{h}^{n} \cdot \nabla \mathbf{u}_{h}^{n}, \mathbf{u}_{h}^{n+1}\right)+\frac{1}{2}\left(\nabla \cdot \boldsymbol{J}_{h}^{n} \mathbf{u}_{h}^{n}, \mathbf{u}_{h}^{n+1}\right)\right) .
\end{aligned}
$$

By combining (3.55)-(3.60), we derive

$$
\begin{aligned}
\left\|\sigma_{h}^{n+1} \mathbf{u}_{h}^{n+1}\right\|^{2} & -\left\|\sigma_{h}^{n} \mathbf{u}_{h}^{n}\right\|^{2}+\frac{\delta t^{2}}{\chi}\left(\left\|\nabla p_{h}^{n+1}\right\|^{2}-\left\|\nabla p_{h}^{n}\right\|^{2}\right)+\frac{\delta t^{2}}{\chi}\left\|\nabla p_{h}^{n}-\nabla p_{h}^{n-1}\right\|^{2} \\
& +\lambda \epsilon\left(\left\|\nabla \phi_{h}^{n+1}\right\|^{2}-\left\|\nabla \phi_{h}^{n}\right\|^{2}+\left\|\nabla \phi_{h}^{n+1}-\nabla \phi_{h}^{n}\right\|^{2}\right) \\
& +\lambda \frac{s}{\epsilon}\left(\left\|\phi_{h}^{n+1}\right\|^{2}-\left\|\phi_{h}^{n}\right\|^{2}+\left\|\phi_{h}^{n+1}-\phi_{h}^{n}\right\|^{2}\right) \\
& +\frac{2 \lambda}{\epsilon}\left(\left|U^{n+1}\right|^{2}-\left|U^{n}\right|^{2}+\left|U^{n+1}-U^{n}\right|^{2}\right) \\
& +\left(\left|Q^{n+1}\right|^{2}-\left|Q^{n}\right|^{2}+\left|Q^{n+1}-Q^{n}\right|^{2}\right) \\
& +\left(\left|R^{n+1}\right|^{2}-\left|R^{n}\right|^{2}+\left|R^{n+1}-R^{n}\right|^{2}\right) \\
& \leq-2 M \delta t\left\|\nabla \mu_{h}^{n+1}\right\|^{2}-\delta t\left\|\sqrt{\nu_{h}^{n+1}} D\left(\mathbf{u}_{h}^{n+1}\right)\right\|^{2} .
\end{aligned}
$$

Finally, we obtain (3.39) from (3.61) after dropping some unnecessary positive terms. 
Remark 3.6. The energy stability is formally derived for the numerical scheme developed in this paper. Since energy stability guarantees the stability of $\phi^{n+1}$ in $H^{1}$, it is expected that the relevant error estimates follow the same reasoning method. Following the error analysis carried out for the different scheme of the Navier-Stokes coupled Cahn-Hilliard phase-field model for the constant density case, $c f$. [6], the error analysis associated with the developed scheme in this article will be performed in our future work.

\subsection{Scheme implementation}

By using the nonlocal property of the auxiliary variables $U, Q, R$, we now present a novel decoupling implementations of the scheme (3.29)-(3.36). Since (3.36) is the Poisson equation with constant coefficients, we only need to discuss the steps 1 and 2 .

Implementation of step 1. We use the nonlocal property of $Q^{n+1}$ and $U^{n+1}$ to solve the $\phi_{h}^{n+1}, \mu_{h}^{n+1}, \tilde{\mathbf{u}}_{h}^{n+1}$, $U^{n+1}$ in step 1.

First, using $Q^{n+1}$, the variables $\phi_{h}^{n+1}, \mu_{h}^{n+1}, \tilde{\mathbf{u}}_{h}^{n+1}, U^{n+1}$ are split into the following linear combination form

$$
\begin{cases}\phi_{h}^{n+1}=\phi_{1 h}^{n+1}+Q^{n+1} \phi_{2 h}^{n+1}, & \mu_{h}^{n+1}=\mu_{1 h}^{n+1}+Q^{n+1} \mu_{2 h}^{n+1}, \\ \tilde{\mathbf{u}}_{h}^{n+1}=\tilde{\mathbf{u}}_{1 h}^{n+1}+Q^{n+1} \tilde{\mathbf{u}}_{2 h}^{n+1}, & U^{n+1}=U_{1}^{n+1}+Q^{n+1} U_{2}^{n+1} .\end{cases}
$$

Then the scheme (3.29), (3.30) and (3.32) can be written as

$$
\left\{\begin{aligned}
&\left(\frac{\phi_{1 h}^{n+1}+Q^{n+1} \phi_{2 h}^{n+1}-\phi_{h}^{n}}{\delta t},\right.\left.w_{h}\right)-Q^{n+1}\left(\mathbf{u}_{h}^{n} \phi_{h}^{n}, \nabla w_{h}\right) \\
&=-M\left(\nabla\left(\mu_{1 h}^{n+1}+Q^{n+1} \mu_{2 h}^{n+1}\right), \nabla w_{h}\right), \\
&\left(\mu_{1 h}^{n+1}+Q^{n+1} \mu_{2 h}^{n+1}, \psi_{h}\right)= \lambda \epsilon\left(\nabla\left(\phi_{1 h}^{n+1}+Q^{n+1} \phi_{2 h}^{n+1}\right), \nabla \psi_{h}\right) \\
&+\frac{\lambda}{\epsilon}\left(U_{1}^{n+1}+Q^{n+1} U_{2}^{n+1}\right)\left(H_{h}^{n}, \psi_{h}\right) \\
&+\lambda \frac{s}{\epsilon}\left(\phi_{1 h}^{n+1}+Q^{n+1} \phi_{2 h}^{n+1}, \psi_{h}\right), \\
&\left(\rho_{h}^{n} \frac{\tilde{\mathbf{u}}_{1 h}^{n+1}+Q^{n+1} \tilde{\mathbf{u}}_{2 h}^{n+1}-\mathbf{u}_{h}^{n}}{\delta t}, \boldsymbol{v}_{h}\right)+Q^{n+1}\left(\phi_{h}^{n} \nabla \mu_{h}^{n}, \boldsymbol{v}_{h}\right)=0 .
\end{aligned}\right.
$$

According to $Q^{n+1}$, we split the system (3.63) into the following two sub-systems:

$$
\begin{aligned}
& \left\{\begin{array}{l}
\left(\frac{\phi_{1 h}^{n+1}}{\delta t}, w_{h}\right)+M\left(\nabla \mu_{1 h}^{n+1}, \nabla w_{h}\right)=\left(\frac{\phi_{h}^{n}}{\delta t}, w_{h}\right) \\
\left(\mu_{1 h}^{n+1}, \psi_{h}\right)=\lambda \epsilon\left(\nabla \phi_{1 h}^{n+1}, \nabla \psi_{h}\right)+\frac{\lambda}{\epsilon} U_{1}^{n+1}\left(H_{h}^{n}, \psi_{h}\right)+\lambda \frac{s}{\epsilon}\left(\phi_{1 h}^{n+1}, \psi_{h}\right) \\
\left(\tilde{\mathbf{u}}_{1 h}^{n+1}, \boldsymbol{v}_{h}\right)=\left(\mathbf{u}_{h}^{n}, \boldsymbol{v}_{h}\right)
\end{array}\right. \\
& \left\{\begin{array}{l}
\left(\frac{\phi_{2 h}^{n+1}}{\delta t}, w_{h}\right)+M\left(\nabla \mu_{2 h}^{n+1}, \nabla w_{h}\right)=\left(\mathbf{u}_{h}^{n} \phi_{h}^{n}, \nabla w_{h}\right), \\
\left(\mu_{2 h}^{n+1}, \psi_{h}\right)=\lambda \epsilon\left(\nabla \phi_{2 h}^{n+1}, \nabla \psi_{h}\right)+\frac{\lambda}{\epsilon} U_{2}^{n+1}\left(H_{h}^{n}, \psi_{h}\right)+\lambda \frac{s}{\epsilon}\left(\phi_{2 h}^{n+1}, \psi_{h}\right) \\
\left(\rho_{h}^{n} \frac{\tilde{\mathbf{u}}_{2 h}^{n+1}}{\delta t}, \boldsymbol{v}_{h}\right)+\left(\phi_{h}^{n} \nabla \mu_{h}^{n}, \boldsymbol{v}_{h}\right)=0
\end{array}\right.
\end{aligned}
$$

We continue to apply the nonlocal property of the variable $U_{1}^{n+1}$ and $U_{2}^{n+1}$ and the splitting technique to solve (3.64) and (3.65). Namely, the four variables $\phi_{1 h}^{n+1}, \mu_{1 h}^{n+1}, \phi_{2 h}^{n+1}, \mu_{2 h}^{n+1}$ are further written as the following linear 
split form consisting the nonlocal variables $U_{1}^{n+1}$ an $U_{2}^{n+1}$, respectively, i.e.,

$$
\begin{cases}\phi_{1 h}^{n+1}=\phi_{11 h}^{n+1}+U_{1}^{n+1} \phi_{12 h}^{n+1}, & \mu_{1 h}^{n+1}=\mu_{11 h}^{n+1}+U_{1}^{n+1} \mu_{12 h}^{n+1}, \\ \phi_{2 h}^{n+1}=\phi_{21 h}^{n+1}+U_{2}^{n+1} \phi_{22 h}^{n+1}, & \mu_{2 h}^{n+1}=\mu_{21 h}^{n+1}+U_{2}^{n+1} \mu_{22 h}^{n+1} .\end{cases}
$$

We replace $\phi_{1 h}^{n+1}, \mu_{1 h}^{n+1}, \phi_{2 h}^{n+1}, \mu_{2 h}^{n+1}$ in (3.64) and (3.65) by using (3.66), and the split the obtained results according to $U_{1}^{n+1}$ and $U_{2}^{n+1}$. Thus we get the following four sub-systems:

$$
\begin{aligned}
& \left\{\begin{array}{l}
\left(\frac{\phi_{11 h}^{n+1}}{\delta t}, w_{h}\right)+M\left(\nabla \mu_{11 h}^{n+1}, \nabla w_{h}\right)=\left(\frac{\phi_{h}^{n}}{\delta t}, w_{h}\right), \\
\left(\mu_{11 h}^{n+1}, \psi_{h}\right)=\lambda \epsilon\left(\nabla \phi_{11 h}^{n+1}, \nabla \psi_{h}\right)+\lambda \frac{s}{\epsilon}\left(\phi_{11 h}^{n+1}, \psi_{h}\right),
\end{array}\right. \\
& \left\{\begin{array}{l}
\left(\frac{\phi_{12 h}^{n+1}}{\delta t}, w_{h}\right)+M\left(\nabla \mu_{12 h}^{n+1}, \nabla w_{h}\right)=0 \\
\left(\mu_{12 h}^{n+1}, \psi_{h}\right)=\lambda \epsilon\left(\nabla \phi_{12 h}^{n+1}, \nabla \psi_{h}\right)+\lambda \frac{s}{\epsilon}\left(\phi_{12 h}^{n+1}, \psi_{h}\right)+\frac{\lambda}{\epsilon}\left(H_{h}^{n}, \psi_{h}\right),
\end{array}\right. \\
& \left\{\begin{array}{l}
\left(\frac{\phi_{21 h}^{n+1}}{\delta t}, w_{h}\right)+M\left(\nabla \mu_{21 h}^{n+1}, \nabla w_{h}\right)=\left(\mathbf{u}_{h}^{n} \phi_{h}^{n}, \nabla w_{h}\right), \\
\left(\mu_{21 h}^{n+1}, \psi_{h}\right)=\lambda \epsilon\left(\nabla \phi_{21 h}^{n+1}, \nabla \psi_{h}\right)+\lambda \frac{s}{\epsilon}\left(\phi_{21 h}^{n+1}, \psi_{h}\right),
\end{array}\right. \\
& \left\{\begin{array}{l}
\left(\frac{\phi_{22 h}^{n+1}}{\delta t}, w_{h}\right)+M\left(\nabla \mu_{22 h}^{n+1}, \nabla w_{h}\right)=0, \\
\left(\mu_{22 h}^{n+1}, \psi_{h}\right)=\lambda \epsilon\left(\nabla \phi_{22 h}^{n+1}, \nabla \psi_{h}\right)+\lambda \frac{s}{\epsilon}\left(\phi_{22 h}^{n+1}, \psi_{h}\right)+\frac{\lambda}{\epsilon}\left(H_{h}^{n}, \psi_{h}\right) .
\end{array}\right.
\end{aligned}
$$

The unknowns $\phi_{11 h}^{n+1}, \phi_{12 h}^{n+1}, \phi_{21 h}^{n+1}, \phi_{22 h}^{n+1}, \mu_{11 h}^{n+1}, \mu_{12 h}^{n+1}, \mu_{21 h}^{n+1}, \mu_{22 h}^{n+1}$ can be easily solved from the above four subsystems (3.67)-(3.70), since all coefficients are constants and all nonlinear terms are given explicitly (Note that (3.68) and (3.70) are the same, so $\phi_{12 h}^{n+1}=\phi_{22 h}^{n+1}$ and $\left.\mu_{12 h}^{n+1}=\mu_{22 h}^{n+1}\right)$.

Second, we solve the nonlocal unknown variables $U_{1}^{n+1}$ and $U_{2}^{n+1}$. Using the split form of $U^{n+1}$ given in (3.62), we rewrite (3.31) as

$$
U_{1}^{n+1}+Q^{n+1} U_{2}^{n+1}=\frac{1}{2}\left(H_{h}^{n}, \phi_{1 h}^{n+1}+Q^{n+1} \phi_{2 h}^{n+1}\right)+g^{n},
$$

where $g^{n}=U^{n}-\frac{1}{2}\left(H_{h}^{n}, \phi_{h}^{n}\right)$ is an explicit known term. Then, according to $Q^{n+1}$, we decompose (3.71) into the following two equations:

$$
\left\{\begin{aligned}
U_{1}^{n+1} & =\frac{1}{2}\left(H_{h}^{n}, \phi_{1 h}^{n+1}\right)+g^{n} \\
U_{2}^{n+1} & =\frac{1}{2}\left(H_{h}^{n}, \phi_{2 h}^{n+1}\right)
\end{aligned}\right.
$$

We replace $\phi_{1 h}^{n+1}, \phi_{2 h}^{n+1}$ by using the variables $\phi_{11 h}^{n+1}, \phi_{12 h}^{n+1}, \phi_{21 h}^{n+1}, \phi_{22 h}^{n+1}, U_{1}^{n+1}, U_{2}^{n+1}$ given (3.66), we obtain

$$
\left\{\begin{array}{l}
U_{1}^{n+1}=\frac{1}{2}\left(H_{h}^{n}, \phi_{11 h}^{n+1}+U_{1}^{n+1} \phi_{12 h}^{n+1}\right)+g^{n}, \\
U_{2}^{n+1}=\frac{1}{2}\left(H_{h}^{n}, \phi_{21 h}^{n+1}+U_{2}^{n+1} \phi_{22 h}^{n+1}\right) .
\end{array}\right.
$$


We factorize (3.73) to get $U_{1}^{n+1}, U_{2}^{n+1}$ that read as follows:

$$
\left\{\begin{array}{l}
U_{1}^{n+1}=\frac{\frac{1}{2}\left(H_{h}^{n}, \phi_{11 h}^{n+1}\right)+g^{n}}{1-\frac{1}{2}\left(H_{h}^{n}, \phi_{12 h}^{n+1}\right)} \\
U_{2}^{n+1}=\frac{\frac{1}{2}\left(H_{h}^{n}, \phi_{21 h}^{n+1}\right)}{1-\frac{1}{2}\left(H_{h}^{n}, \phi_{22 h}^{n+1}\right)}
\end{array}\right.
$$

The solvability of the nonlocal unknown variables $U_{1}^{n+1}, U_{2}^{n+1}$ can be shown by verifying that the denominators in (3.74) are non-zero, which can be obtained by applying a simple energy estimate. For (3.68), we set $w_{h}=$ $-\delta t \mu_{12 h}^{n+1}, \psi_{h}=\phi_{12 h}^{n+1}$, and combine the two obtained equations to get

$$
-\frac{\lambda}{\epsilon}\left(H_{h}^{n}, \phi_{12 h}^{n+1}\right)=\lambda \epsilon\left\|\nabla \phi_{12 h}^{n+1}\right\|^{2}+\frac{\lambda s}{\epsilon}\left\|\phi_{12 h}^{n+1}\right\|^{2}+M \delta t\left\|\nabla \mu_{12 h}^{n+1}\right\|^{2} \geq 0 .
$$

This means $1-\frac{1}{2}\left(H_{h}^{n}, \phi_{12 h}^{n+1}\right) \neq 0$. From $\phi_{12 h}^{n+1}=\phi_{22 h}^{n+1}$, we conclude that $U_{1}^{n+1}, U_{2}^{n+1}$ are always solvable. Once $U_{1}^{n+1}, U_{2}^{n+1}$ are obtained, we can get $\phi_{1 h}^{n+1}, \phi_{2 h}^{n+1}, \mu_{1 h}^{n+1}, \mu_{2 h}^{n+1}$ from (3.66).

Third, we solve the nonlocal unknown variable $Q^{n+1}$ from (3.33). Using the split form of $\mu_{h}^{n+1}$ and $\tilde{\mathbf{u}}_{h}^{n+1}$ given in (3.62), we rewrite (3.33) as:

$$
\left(\frac{1}{\delta t}-\theta_{2}\right) Q^{n+1}=\frac{Q^{n}}{\delta t}+\theta_{1}
$$

where

$$
\left\{\begin{array}{l}
\theta_{1}=-\left(\mathbf{u}_{h}^{n} \phi_{h}^{n}, \nabla \mu_{1 h}^{n+1}\right)+\left(\phi_{h}^{n} \nabla \mu_{h}^{n}, \tilde{\mathbf{u}}_{1 h}^{n+1}\right), \\
\theta_{2}=-\left(\mathbf{u}_{h}^{n} \phi_{h}^{n}, \nabla \mu_{2 h}^{n+1}\right)+\left(\phi_{h}^{n} \nabla \mu_{h}^{n}, \tilde{\mathbf{u}}_{2 h}^{n+1}\right) .
\end{array}\right.
$$

The solvability of the nonlocal unknown variable $Q^{n+1}, U_{2}^{n+1}$ can be verified by showing $\frac{1}{\delta t}-\theta_{2} \neq 0$. By taking $w_{h}=\mu_{2 h}^{n+1}, \psi_{h}=-\frac{1}{\delta t} \phi_{2 h}^{n+1}$ in (3.65), and adding up the obtained two equations, we get

$$
\left(\mathbf{u}_{h}^{n} \phi_{h}^{n}, \nabla \mu_{2 h}^{n+1}\right)=M\left\|\nabla \mu_{2 h}^{n+1}\right\|^{2}+\frac{\lambda \epsilon}{\delta t}\left\|\nabla \phi_{2 h}^{n+1}\right\|^{2}+\frac{\lambda s}{\delta t \epsilon}\left\|\phi_{2 h}^{n+1}\right\|^{2}+\frac{\lambda}{\epsilon \delta t} U_{2}^{n+1}\left(H_{h}^{n}, \phi_{2 h}^{n+1}\right) .
$$

From the second equation in (3.72), we immediately get

$$
U_{2}^{n+1}\left(H_{h}^{n}, \phi_{2 h}^{n+1}\right)=\frac{1}{2}\left(H_{h}^{n}, \phi_{2 h}^{n+1}\right)^{2},
$$

which implies

$$
\left(\mathbf{u}_{h}^{n} \phi_{h}^{n}, \nabla \mu_{2 h}^{n+1}\right) \geq 0 .
$$

By taking $\boldsymbol{v}_{h}=\tilde{\mathbf{u}}_{2 h}^{n+1}$ in (3.65) and using (3.38), we derive

$$
-\left(\phi_{h}^{n} \nabla \mu_{h}^{n}, \tilde{\mathbf{u}}_{2 h}^{n+1}\right)=\frac{1}{\delta t} \int_{\Omega} \rho_{h}^{n}\left|\tilde{\mathbf{u}}_{2 h}^{n+1}\right|^{2} \mathrm{~d} \boldsymbol{x} \geq 0 .
$$

Using (3.80) and (3.81), we derive $-\theta_{2} \geq 0$, that implies (3.76) is solvable. Then, once $Q^{n+1}$ is obtained from (3.76), we update $\phi_{h}^{n+1}, \mu_{h}^{n+1}, \tilde{\mathbf{u}}_{h}^{n+1}, U^{n+1}$ from (3.62), which finishes step 1 .

Implementation of step 2: We now give the detailed implementation process of the scheme (3.34) and (3.35) in step 2. 
First, by using the nonlocal variable $R^{n+1}$, the unknown velocity field $\mathbf{u}_{h}^{n+1}$ can be written as a linear split form that reads as

$$
\mathbf{u}_{h}^{n+1}=\mathbf{u}_{1 h}^{n+1}+R^{n+1} \mathbf{u}_{2 h}^{n+1} .
$$

We replace $\mathbf{u}_{h}^{n+1}$ using (3.82) and then decompose the obtained equation into the following two sub-equations according to $R^{n+1}$, namely,

$$
\left\{\begin{array}{c}
\left(\frac{1}{2 \delta t}\left(\rho_{h}^{n+1}+\rho_{h}^{n}\right) \mathbf{u}_{1 h}^{n+1}, \boldsymbol{v}_{h}\right)+\frac{1}{2}\left(\nu_{h}^{n+1} D\left(\mathbf{u}_{1 h}^{n+1}\right), D\left(\boldsymbol{v}_{h}\right)\right)=\left(\frac{\rho_{h}^{n}}{\delta t} \tilde{\mathbf{u}}_{h}^{n+1}-\nabla\left(2 p_{h}^{n}-p_{h}^{n-1}\right), \boldsymbol{v}_{h}\right) \\
\left(\frac{1}{2 \delta t}\left(\rho_{h}^{n+1}+\rho_{h}^{n}\right) \mathbf{u}_{2 h}^{n+1}, \boldsymbol{v}_{h}\right)+\frac{1}{2}\left(\nu_{h}^{n+1} D\left(\mathbf{u}_{2 h}^{n+1}\right), D\left(\boldsymbol{v}_{h}\right)\right)=-\left(\rho_{h}^{n}\left(\mathbf{u}_{h}^{n} \cdot \nabla\right) \mathbf{u}_{h}^{n}, \boldsymbol{v}_{h}\right) \\
-\frac{1}{2}\left(\nabla \cdot\left(\rho_{h}^{n} \mathbf{u}^{n}\right) \mathbf{u}_{h}^{n}, \boldsymbol{v}_{h}\right)-\left(\boldsymbol{J}_{h}^{n} \cdot \nabla \mathbf{u}_{h}^{n}, \boldsymbol{v}_{h}\right)-\frac{1}{2}\left(\nabla \cdot \boldsymbol{J}_{h}^{n} \mathbf{u}_{h}^{n}, \boldsymbol{v}_{h}\right) .
\end{array}\right.
$$

Note that the variable coefficients $\rho_{h}^{n+1}+\rho_{h}^{n}$ and $\nu_{h}^{n+1}$ are all positive from (3.38) due to the application of the cut-off technique, which implies these variable-coefficient equations are well-posed. After solving these variable-coefficient elliptic equations, we obtain $\mathbf{u}_{1 h}^{n+1}, \mathbf{u}_{2 h}^{n+1}$.

Second, we solve the auxiliary variable $R^{n+1}$ from (3.35). By applying the split form of the variable $\mathbf{u}_{h}^{n+1}$ given in (3.82) to (3.35), we obtain the following formulation:

$$
\left(\frac{1}{\delta t}-\eta_{2}\right) R^{n+1}=\frac{1}{\delta t} R^{n}+\eta_{1}
$$

where

$$
\begin{aligned}
\eta_{i}= & \left(\rho_{h}^{n}\left(\mathbf{u}_{h}^{n} \cdot \nabla\right) \mathbf{u}_{h}^{n}, \mathbf{u}_{i h}^{n+1}\right)+\frac{1}{2}\left(\nabla \cdot\left(\rho_{h}^{n} \mathbf{u}_{h}^{n}\right) \mathbf{u}_{h}^{n}, \mathbf{u}_{i h}^{n+1}\right)+\left(\boldsymbol{J}_{h}^{n} \cdot \nabla \mathbf{u}_{h}^{n}, \mathbf{u}_{i h}^{n+1}\right) \\
& +\frac{1}{2}\left(\nabla \cdot \boldsymbol{J}_{h}^{n} \mathbf{u}_{h}^{n}, \mathbf{u}_{i h}^{n+1}\right), i=1,2
\end{aligned}
$$

The solvability of (3.84) can be verified by showing $\frac{1}{\delta t}-\eta_{2} \neq 0$, which is obtained by the energy estimate. By taking $\boldsymbol{v}_{h}=\mathbf{u}_{2 h}^{n+1}$ in the second equation of (3.83) and using (3.38), we derive

$$
-\eta_{2}=\frac{1}{2 \delta t} \int_{\Omega}\left(\rho_{h}^{n+1}+\rho_{h}^{n}\right)\left|\mathbf{u}_{2 h}^{n+1}\right|^{2} \mathrm{~d} \boldsymbol{x}+\frac{1}{2} \int_{\Omega} \nu_{h}^{n+1}\left|D\left(\mathbf{u}_{2 h}^{n+1}\right)\right|^{2} \mathrm{~d} \boldsymbol{x} \geq 0,
$$

that implies (3.84) is solvable. Once $R^{n+1}$ is computed, we update $\mathbf{u}_{h}^{n+1}$ from (3.82).

Hence, the total cost at each time step only includes three constant-coefficient elliptic systems ((3.67)-(3.70)); two variable coefficient elliptic equations (3.83); and one pressure Poisson equation (3.36). It can be seen that each step is the realization of decoupling type, which means that the calculation is

Remark 3.7. We recall a fully-decoupled type scheme has been developed in $[4,25,33]$. Here, we give the detailed scheme so that we can compare the scheme developed in $[4,25,33]$ and the scheme $(3.29)-(3.36)$ developed in this article. For simplicity, we only discretize the related terms while other terms remain continuously so that the readers can see the detailed discretization more clearly.

First, compared with (3.32), the scheme given in $[4,25,33]$ discretizes it as

$$
\rho^{n} \frac{\tilde{\mathbf{u}}^{n+1}-\mathbf{u}^{n}}{\delta t}+\phi^{n} \underbrace{\nabla \mu^{n+1}}_{\text {implicit }}=0 .
$$


Therefore, we get

$$
\tilde{\mathbf{u}}^{n+1}=\mathbf{u}^{n}-\delta t \frac{1}{\rho^{n}} \phi^{n} \nabla \mu^{n+1} .
$$

The term $\tilde{\mathbf{u}}^{n+1}$ is used as the advective velocity in the Cahn-Hilliard equation which is discretized as

$$
\frac{\phi^{n+1}-\phi^{n}}{\delta t}+\nabla \cdot\left(\tilde{\mathbf{u}}^{n+1} \phi^{n}\right)=M \Delta \mu^{n+1} .
$$

By replacing $\tilde{\mathbf{u}}^{n+1}$ in the above using (3.87), we get

$$
\frac{\phi^{n+1}-\phi^{n}}{\delta t}+\nabla \cdot\left(\mathbf{u}^{n} \phi^{n}\right)-\delta t \nabla \cdot\left(\frac{1}{\rho^{n}} \phi^{n} \phi^{n} \nabla \mu^{n+1}\right)=M \Delta \mu^{n+1} .
$$

Therefore, the scheme in $[4,25,33]$ leads to solve the Cahn-Hilliard equation with variable coefficients with a factor $\frac{1}{\rho^{n}}$, which is very costly in computational costs.

Second, for the discretization of the nonlinear terms in the momentum equation, compared with (3.34), the scheme in $[4,25,33]$ discretize them as

$$
\rho^{n}\left(\mathbf{u}^{n} \cdot \nabla\right) \mathbf{u}^{n+1}+\frac{1}{2} \nabla \cdot\left(\rho^{n} \mathbf{u}^{n}\right) \mathbf{u}^{n+1}+\boldsymbol{J}^{n} \cdot \nabla \mathbf{u}^{n+1}+\frac{1}{2} \nabla \cdot \boldsymbol{J}^{n} \mathbf{u}^{n+1} .
$$

Although this discrete method can obtain unconditional energy stability, in practice, a large number of variable coefficients bring up unpredictable effects on the condition number of the linear system, leading to higher computational costs. This can be seen in Figure 2c where we compare the average number of iterations per time step needed by our developed scheme (3.29)-(3.36) and the scheme developed in $[4,25,33]$. Some further works on the convergence analysis of the proposed scheme will be performed by following the lines of $[9,10,26]$

\section{Numerical EXAMPLES}

In this section, we carry out the developed algorithm (3.29)-(3.36) using the splitting technique through scalar variables (denoted as DSS for short) to investigate the accuracy and energy stability numerically, and also implement several benchmark problems including the Rayleigh-Taylor instability problems and the dynamics of rising droplets.

We set the computational domain as a rectangular region, and use the Taylor-Hood element (see [14]) for $\boldsymbol{V}_{h}$ and $O_{h}$ that satisfies inf-sup condition and set the finite element spaces (3.20) with $l_{1}=1, l_{2}=2$.

\subsection{Accuracy and stability test}

We perform the $2 \mathrm{D}$ convergence and stability tests. The computational domain is set as $\Omega=[0,2]^{2}$, and the model parameters are set as

$$
\lambda=0.002, \epsilon=0.04, M=1, B=10, \rho_{1}=1000, \rho_{2}=1, \nu_{1}=50, \nu_{2}=1, s=4 .
$$

We set the initial conditions as

$$
\left\{\begin{array}{l}
\phi^{0}(x, y)=1+\sum_{i=1}^{2} \tan h\left(\frac{r_{i}-\sqrt{\left(x-x_{i}\right)^{2}+\left(y-y_{i}\right)^{2}}}{1.5 \epsilon}\right) \\
\mathbf{u}^{0}(x, y)=\mathbf{0}, p^{0}(x, y)=0
\end{array}\right.
$$

where $r_{1}=0.45, r_{2}=0.25, x_{1}=0.6, x_{2}=1.6, y_{1}=0.9, y_{2}=1.2$. The initial profile of $\phi^{0}$ of two circles with different radii is shown in Figure $2 \mathrm{~b}$. 


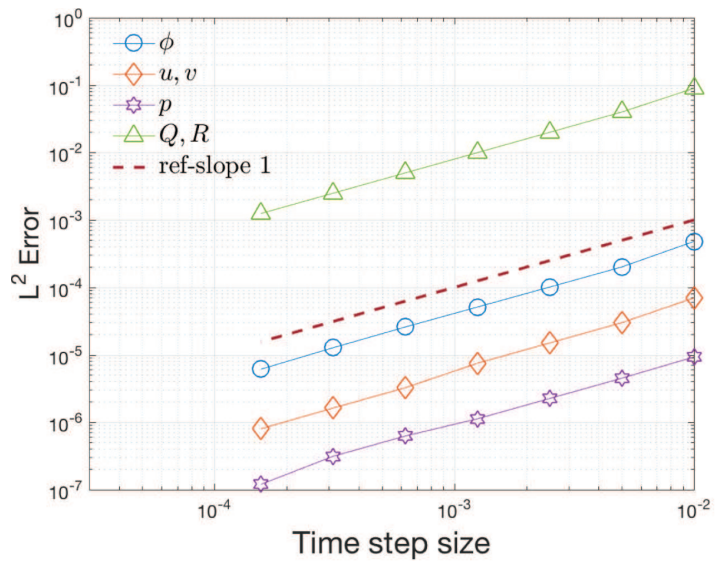

(a)

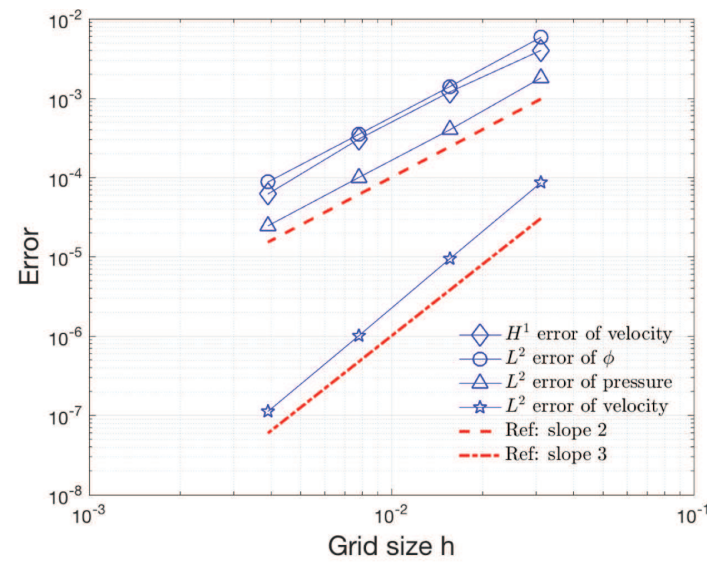

(b)

Figure 1. (a) Convergence tests where the $L^{2}$ numerical errors at $t=0.5$ for the phase-field variable $\phi$, the average of the two velocity components $(u, v)$, the pressure $p$, and the average of the two auxiliary variables $(Q, R)$ are plotted; and (b) convergence order tests for spatial discretization.

To verify the temporal convergence order, we fix mesh size $h=\frac{1}{256}$ so that the grid size is sufficiently small and the spatial discretization errors are negligible compared with the time discretization error. Since the exact solutions are not known, we choose the numerical solutions obtained with a very tiny time step size $\delta t=1 e-9$ computed by the scheme DSS as the exact solution approximately for computing errors. In Figure 1a, the $L^{2}$ errors between the numerical solution and the exact solution at $t=0.5$ are plotted (the exact solution of $Q$ and $R$ is 1 ). We observe that all variables follow the first-order time accuracy.

To verify the spatial convergence order, in Figure 1b, we plot the errors of DSS for various spatial grid size $h$. We choose $\delta t$ sufficiently small $(\delta t=1 e-9)$ so that the errors are only dominated by the spatial discretization error. We use the numerical solutions obtained with a very tiny mesh size $\delta t=1 e-9, \frac{1}{h}=\frac{1}{512}$ computed by the scheme DSS as the exact solution approximately for computing errors. The errors in various norms between the numerical solution and the exact solution at $t=0.2$ are plotted. We can see that the second-order convergence rates are followed by the $H^{1}$-error of the velocity, $L^{2}$-error of the pressure $p$, and $L^{2}$-error of the phase-field variable $\phi$, while third-order convergence rates are observed for the $L^{2}$-error of the velocity. These results are in full agreements with the theoretical expectation of accuracy for $P 2 / P 1$ element for $(\mathbf{u}, p)$ and $P 1$ element for $\phi$.

We further perform the energy stability tests by plotting the total free energy (2.13) evolved over time in Figure 2a by using various time steps. The decays of all energy curves illustrates the energy stability. In Figure $2 \mathrm{~b}$, using $\delta t=0.01$, we plot the time evolution of the total free energy (2.13) (in the original form) and (3.40) (in the modified discrete form). The overlapping of these two energy curves show that these two energies are consistent numerically.

In Figure 2c, we compare the average number of iterations per time step needed by DSS and that needed by the scheme developed in $[4,25,33]$ (see Rem. 3.7, denoted by Stab for short). Note that the time of each iteration is basically the same as the time to solve a Poisson equation with constant coefficients. The comparison shows that the efficiency of DSS is much higher than that of Stab, because DSS needs to solve far fewer variablecoefficient equations. In Figure 2d, we compare the $L^{2}$ error of $\phi$ when using various stabilization parameters $s$. When $s=0$, the scheme is not stable for larger time steps, so the error points are missed. When $s=4$, the accuracy curve is not only very close to the results of $s=0$ for small time steps, but also stable for all other tested big time steps, and the entire curve shows the first-order convergence rate. When $s=20$ and $s=100$, 


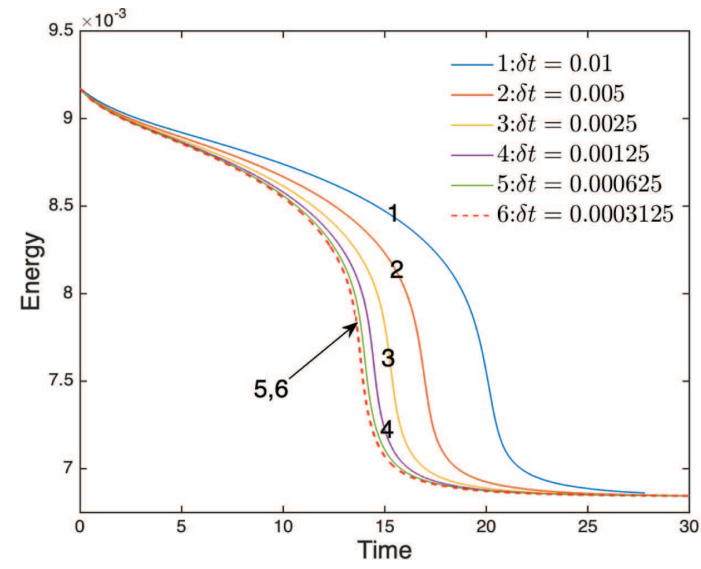

(a)

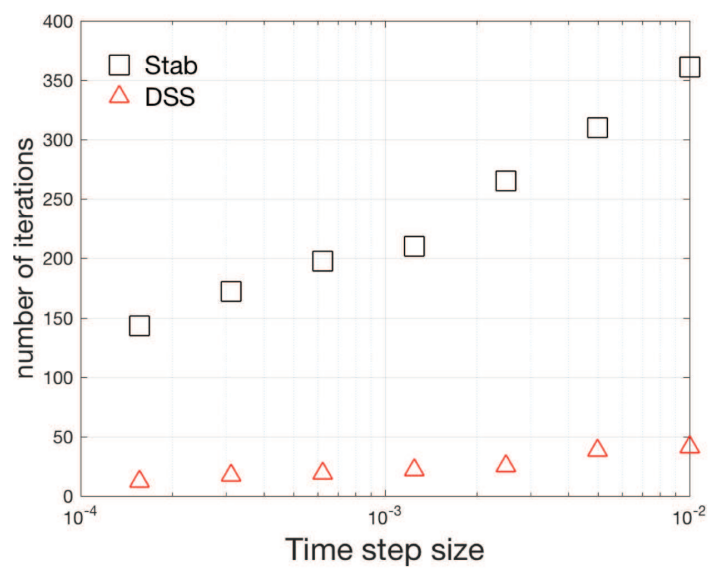

(c)

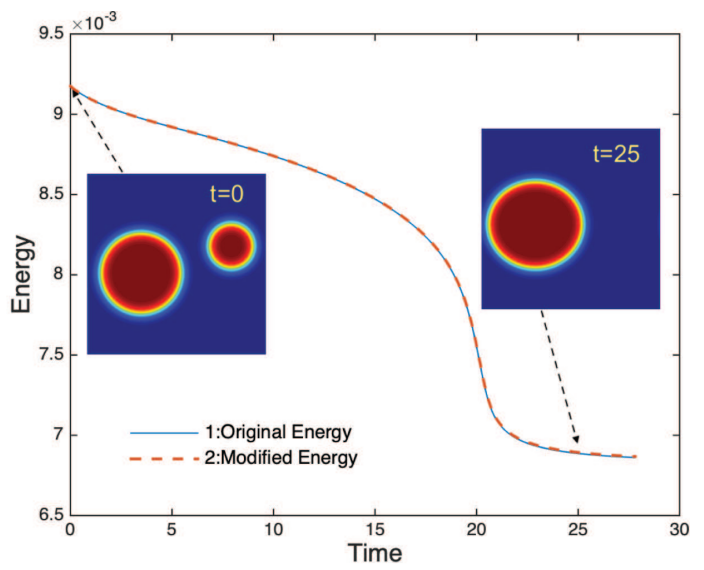

(b)

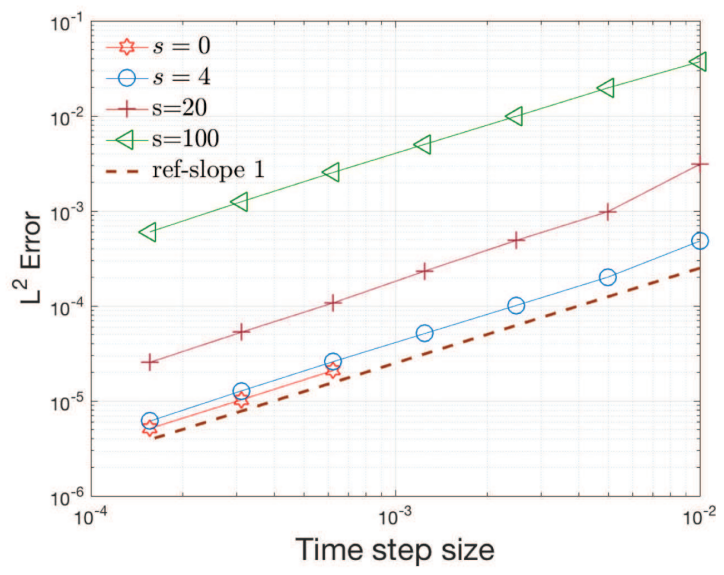

(d)

FiguRE 2. (a) Energy stability tests where the evolutions of the total free energy (2.13) are computed using various time steps; (b) the comparisons of the original energy (2.13) and the modified energy (3.40) computed by using the time step $\delta t=0.01$ (the profiles of $\phi$ at different times are appended); (c) the comparisons of the average number of iterations per time step computed by the schemes DSS and Stab; and (d) the comparisons of $L^{2}$ errors of $\phi$ when using various stabilization parameters $s$.

we can see that even though the scheme is always stable, the accuracy is much worse than the case of $s=4$. Therefore, we adopt $s=4$ in all our computations.

\subsection{Rayleigh-Taylor instability}

Rayleigh-Taylor interface instability is a benchmark phenomenon of two-phase flow with variable density, see $[11,18,24,34]$. The classic experimental setup is that the system consists of two layers of fluids with different densities. Under the action of gravity, slight interference caused by potential energy will cause the heavier fluid to sink, and the lighter fluid will rise, resulting in irregularities (ripples) caused by downward movement will quickly expand into a series of "RT fingers" (shown in Figs. 3 and 5). 

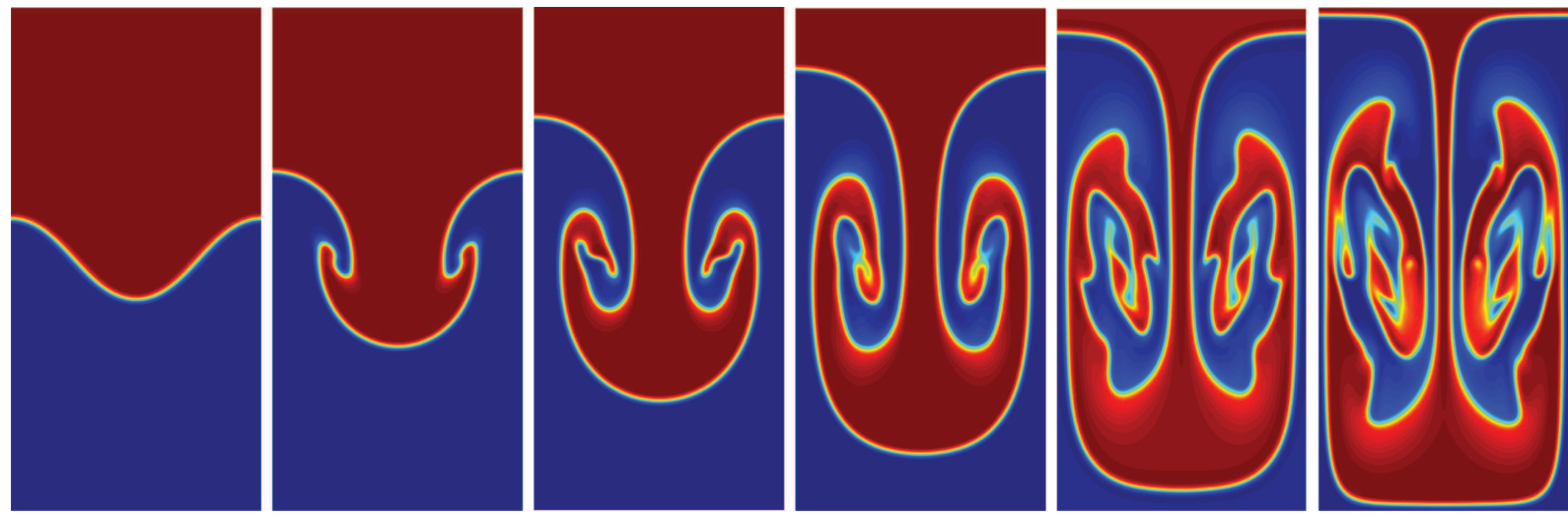

Figure 3. Rayleigh-Taylor instability with Atwood number $A t=0.5$. The profiles of $\phi$ are captured at $t=0.5,1.5,2.5,3.5,4.5$, and 5.5 from left to right.
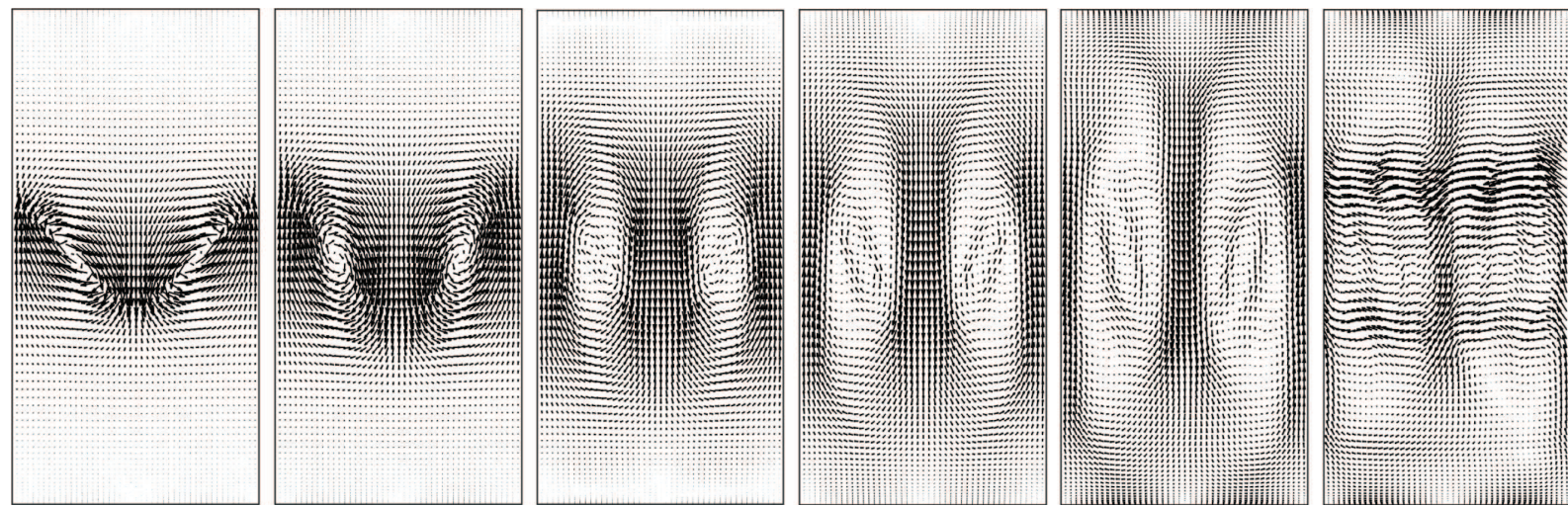

FIgURE 4. The velocity field $\mathbf{u}=(u, v)$ for the Rayleigh-Taylor instability example with $A t=0.5$. Snapshots are taken at $t=0.5,1.5,2.5,3.5,4.5$, and 5.5 from left to right.
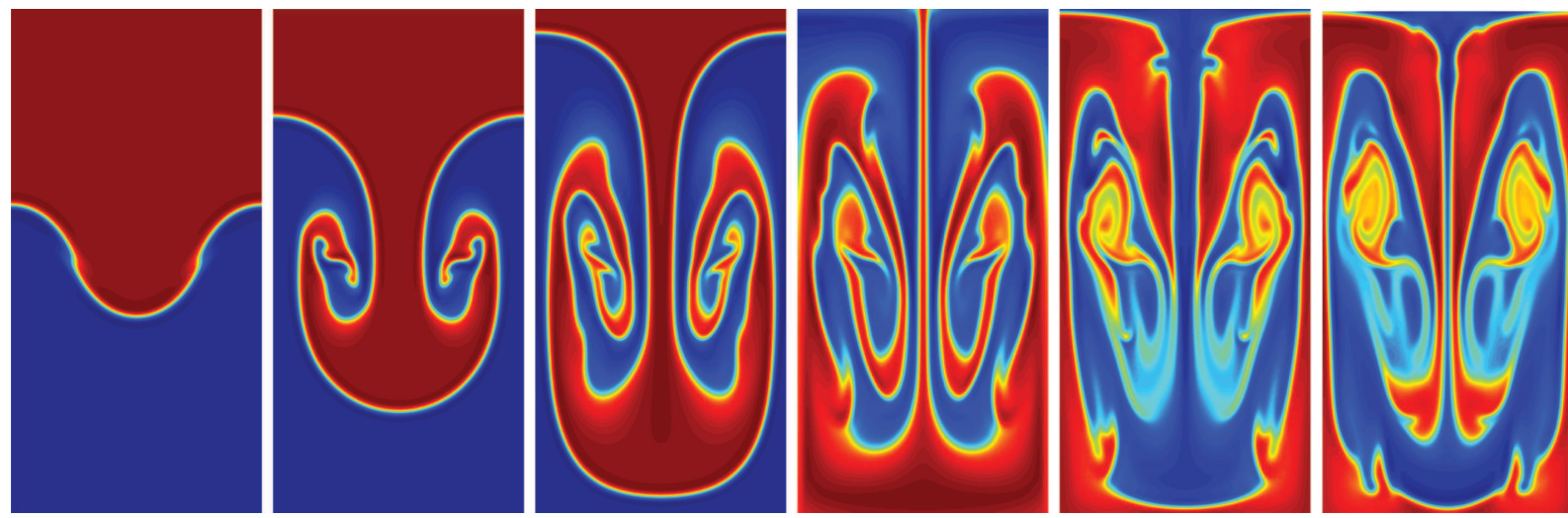

Figure 5. Rayleigh-Taylor instability with Atwood number $A t=0.9802$. Snapshots of $\phi$ are taken at $t=0.5,1.5,2.5,3.5,4.5$, and 5 . 

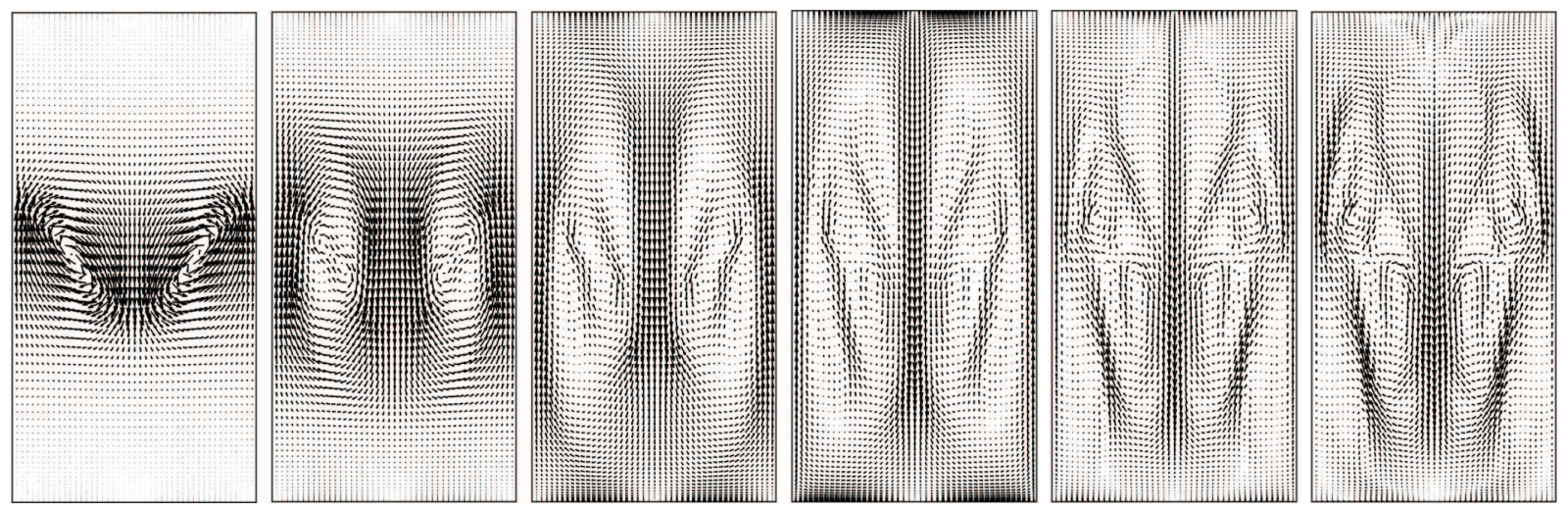

Figure 6 . The velocity field $\mathbf{u}=(u, v)$ for the Rayleigh-Taylor instability example with $A t=0.9802$. Snapshots are taken at $t=0.5,1.5,2.5,3.5,4.5$, and 5 from left to right.

We set the $2 \mathrm{D}$ computational domain as $(x, y) \in \Omega=[0, d] \times[0,2 d]$ and add the gravity field to the momentum equation, which reads as

$$
\rho\left(\mathbf{u}_{t}+(\mathbf{u} \cdot \nabla) \mathbf{u}\right)+\boldsymbol{J}(\mu) \cdot \nabla \mathbf{u}-\nabla \cdot(\nu(\phi) D(\mathbf{u}))+\nabla p+\phi \nabla \mu=\rho \mathbf{g},
$$

where $\mathbf{g}=\left(0, g_{0}\right)$ and $g_{0}$ is the gravity constant. We rescale the variables/parameters by the following way:

$$
\hat{t}=\frac{t}{t_{0}}, \hat{\boldsymbol{x}}=\frac{\boldsymbol{x}}{d_{0}}, \hat{\mathbf{u}}=\frac{\mathbf{u}}{u_{0}}, \hat{\nu}=\frac{\nu}{\sqrt{d_{0}^{3} g_{0}}}, \hat{M}=M, \hat{\lambda}=\lambda, \hat{p}=p, \hat{\rho}=\rho, \hat{\phi}=\phi,
$$

where $t_{0}=\sqrt{d / g_{0}}, u_{0}=\sqrt{d g_{0}}, d_{0}=d$. If the ${ }^{\wedge}$ is omitted, we can see that the dimensionless governing system $(2.2)-(2.3)-(4.3)$ is the same.

We set the initial conditions of a perturbed two-layer fluid as follows,

$$
\phi^{0}(x, y)=\tan h\left(\frac{y-\frac{1}{8} \cos \left(2 \pi \frac{x}{d}\right)-1}{2 \epsilon / 3}\right), \mathbf{u}^{0}=(0,0), p^{0}=0 .
$$

Namely, the heavier fluid with density $\rho_{2}$ is initially set on the top layer, while the light fluid with density $\rho_{1}$ is set below. The model parameters are set as

$$
\begin{aligned}
& d=1, d_{0}=1, g_{0}=10, \lambda=0.001, \epsilon=0.025, M=0.01 \\
& B=10, \rho_{1}=1, \nu_{1}=\nu_{2}=0.001, s=4, g_{0}=1, \delta t=1 e-4, h=\frac{1}{512} .
\end{aligned}
$$

Therefore, the Reynolds number is 1000 .

In the first simulation, we set the density of the heavier fluid as $\rho_{2}=3$ to investigate the dynamical changes of the fluid interface when the density ratio of the two fluids is not much different. The so-called Atwood number $A t$ is given by Tryggvason's definition in [34], which is $A t=\frac{\rho_{\max }-\rho_{\min }}{\rho_{\max }+\rho_{\min }}$. For this case, $A t=0.5$. Snapshots of the profiles of $\phi$ at various times are shown in Figure 3. While the upper heavier fluid flows to the lower layer under the action of the gravity field, a roll-like structure is formed. In the initial stage of the production of the rolling structure, as the main vortex scrolls, some small branches (R-T fingers) are formed, see $t=3.5$, and more complex fingering patterns are produced gradually. We further plot the profiles of the velocity field at various times in Figure 4. 

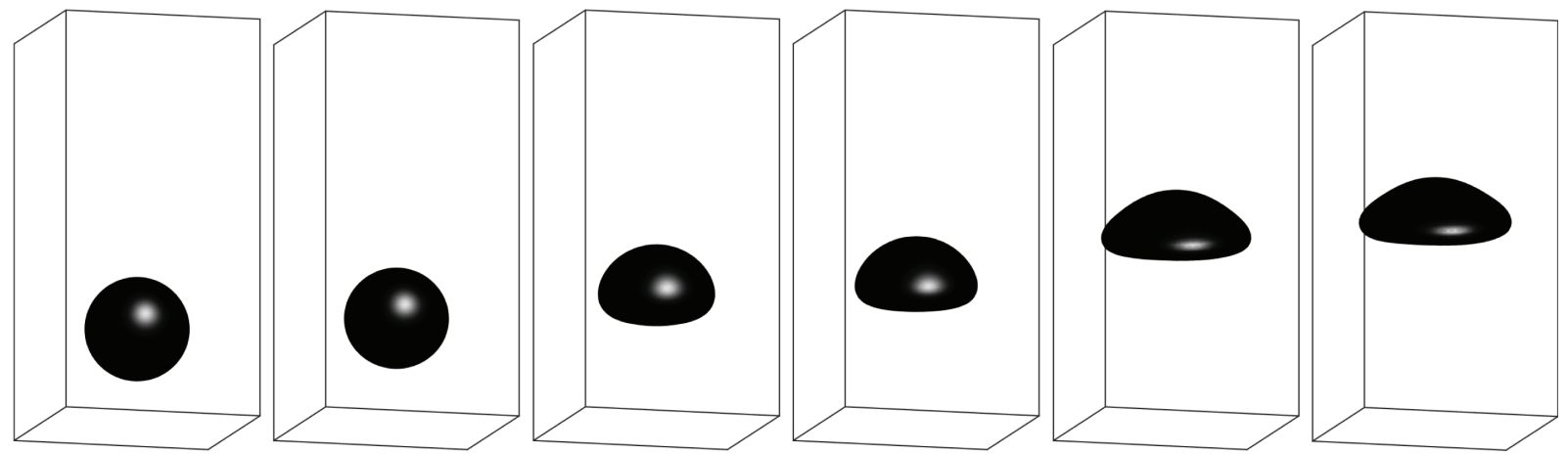

Figure 7. A 3D droplet rising in the gravity field where $\rho_{1}=1$ and $\rho_{2}=1000$. Profiles of the isosurfaces of the interface $\{\phi=0\}$ are taken at $t=0,0.3,0.6,0.7,1.3$, and 1.5.

In the second simulation, we increase the density of the upper heavier fluid as $\rho_{2}=100$, namely, At $=0.9802$. From the snapshots of $\phi$ at different times shown in Figure 5 and the velocity field profiles shown in Figure 6 , we observe that more complex vortices and R-T fingers appear during the formation of the rolling structure. These obtained simulations are qualitatively consistent with numerical simulations and experimental results using different models and different numerical methods, see [11,18, 24,34].

\subsection{Dynamics of rising droplets}

In this numerical example, we examine the rising phenomenon of 3D light droplets immersed in a fluid with a larger density due to the action of the gravitational field. The momentum equation (4.3) equipped with the gravity force $\left(\mathbf{g}=\left(0,0, g_{0}\right)\right)$ is still adopted. The non-dimensionalized method is also consistent with the previous example. We set the computational domain as $\Omega=[0, d] \times[0, d] \times[0, \tilde{d}]$ and the model parameters are set as

$$
\begin{aligned}
& d=1, d_{0}=1, \tilde{d}=2.15, g_{0}=10, \lambda=0.002, \epsilon=0.0175, M=1, B=10, \\
& \rho_{1}=1, \rho_{2}=1000, \nu_{1}=1, \nu_{2}=100, s=4, \delta t=1 e-3, h=\frac{1}{200} .
\end{aligned}
$$

We first study the dynamic changes of the rising deformation of a single droplet. The initial droplet is set at the bottom of the domain with the following formulation:

$$
\begin{aligned}
\phi^{0}(x, y, z) & =\tan h\left(\frac{0.25-\sqrt{(x-0.5)^{2}+(y-0.5)^{2}+(z-0.5)^{2}}}{\epsilon}\right), \\
\mathbf{u}^{0} & =(0,0), p^{0}=0 .
\end{aligned}
$$

Snapshots of the isosurface $\{\phi=0\}$ at different times are plotted in Figure 7. Due to the influence of gravity, the shape of the droplet will change dramatically as it rises gradually, from the initial spherical shape to a flat cap shape. Note that the results of the deformation we obtained are consistent with the physical experimental results given in [3] and the simulation results of different models given in [2].

Next, we set the initial conditions to two non-contact droplets located at different latitudes to study the rising dynamics of multiple droplets under the influence of gravity and examine their mutual influence. The model parameters are still given in (4.7).

In the first simulation, the initial two droplets of the same size are placed at different heights, one of which is suspended directly above the other. This is the so-called coaxial coalescence situation, see [5]. The formulation 

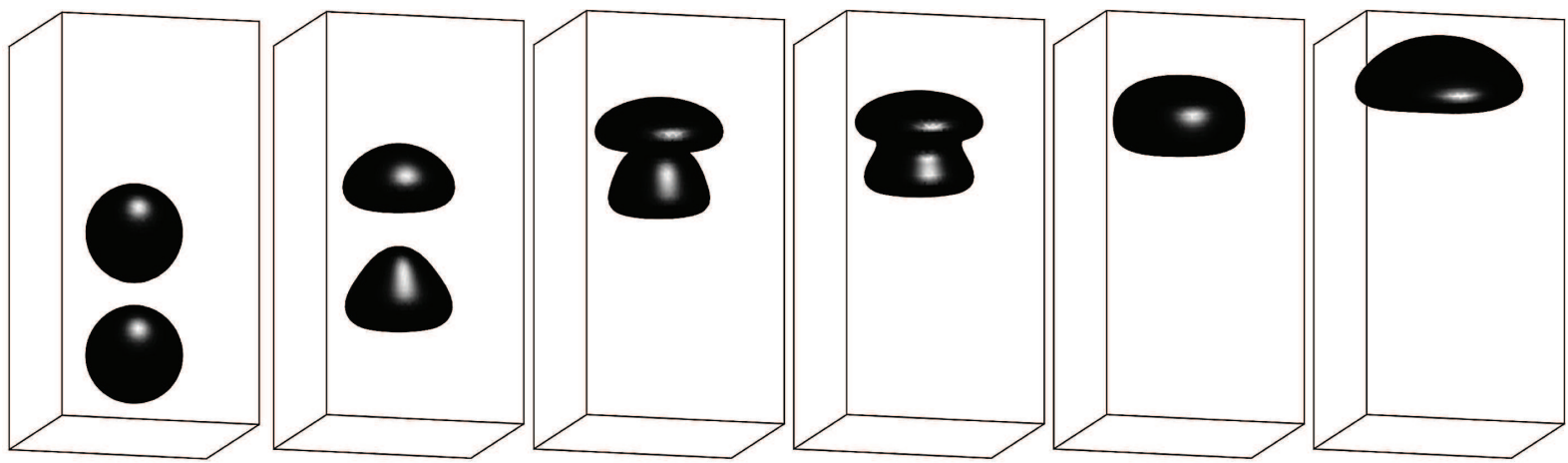

(a)
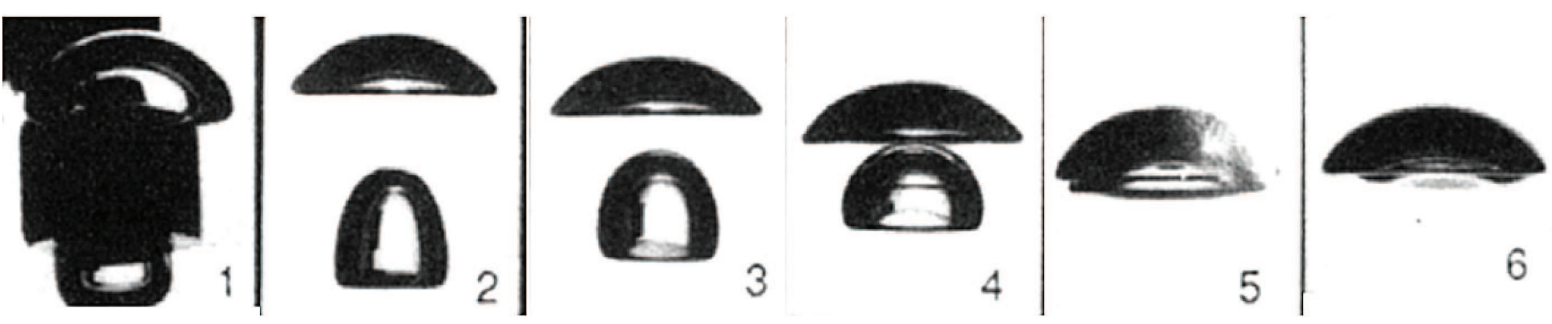

(b)

Figure 8. (a) The dynamical motion of two 3D rising droplets (coaxial coalescence case) in the gravity field where $\rho_{1}=1$ and $\rho_{2}=1000$. Profiles of the isosurfaces of the interface $\{\phi=0\}$ are taken at $t=0,0.7,1.3,1.4,1.6$, and $2.1 ;(\mathrm{b})$ the experimental results by Brereton and Korotney in [5].

of the initial conditions read as follows,

$$
\begin{aligned}
\phi^{0}(x, y, z) & =\sum_{i=1}^{2} \tan h\left(\frac{0.25-\sqrt{\left(x-x_{i}\right)^{2}+\left(y-y_{i}\right)^{2}+\left(z-z_{i}\right)^{2}}}{\epsilon}\right)+1, \\
\mathbf{u}^{0} & =(0,0), p^{0}=0
\end{aligned}
$$

where $x_{1}=x_{2}=y_{1}=y_{2}=0.5, z_{1}=0.4, z_{2}=1.2$.

The isosurface of $\{\phi=0\}$ at different times are shown in Figure 8a, where we can see that the deformations of the upper and lower droplets under the influence of gravity are completely different. At the initial stage, both droplets will change from a spherical shape to a cap shape, but the height of the cap formed by the upper droplet is very short, and the cross section of the cap becomes very large. However, the height of the cap formed by the droplet below is very large, and the cross section becomes relatively small. And the moving speeds of the upper and lower droplets are also different. The lower droplet moves faster, so it will catch up with the higher droplet, and then they merge together (approximately at $t=1.4$ ). For comparison, we show the experimental results of [5] in Figure 8b which can be seen to be very consistent with our numerical simulation qualitatively.

In the second simulation, we also use two droplets of the same size. and place them at different heights, but one droplet is in a staggered position on the other droplet. This is the so-called oblique coalescence case, cf. [5]. The initial conditions are still given in (4.9) with $x_{1}=0.3, x_{2}=0.5$. The isosurface of $\{\phi=0\}$ at different times are plotted in Figure 9a. We can see that when the two droplets start to rise upward, the obvious difference 

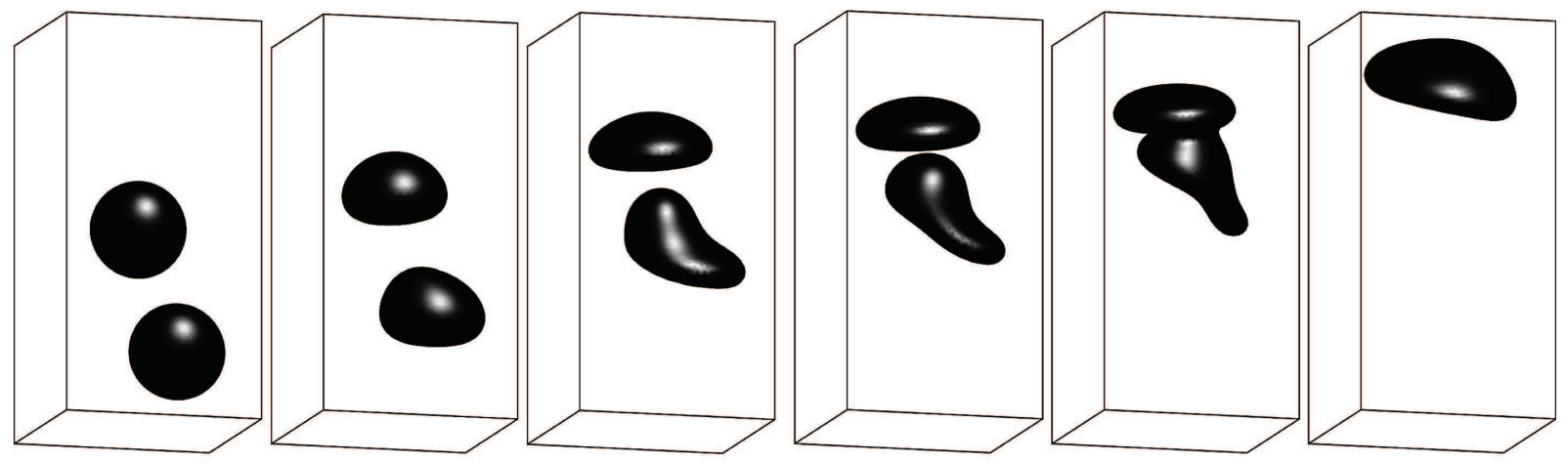

(a)
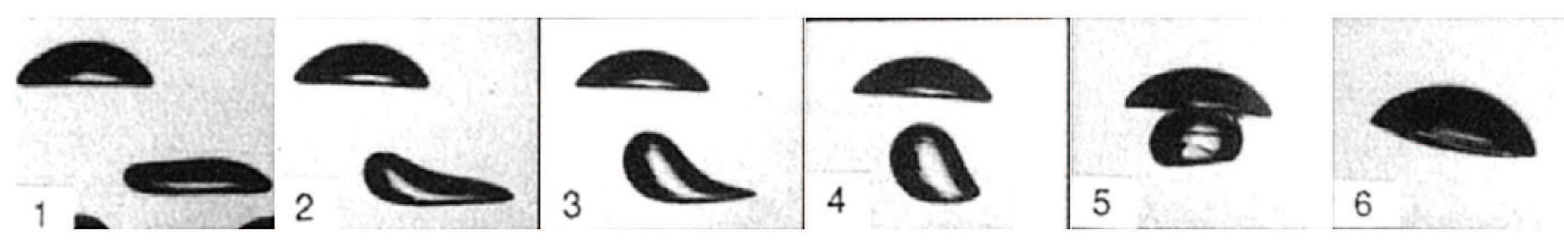

(b)

Figure 9. (a) The dynamical motion of two 3D rising droplets (oblique coalescence case) in the gravity field where $\rho_{1}=1$ and $\rho_{2}=1000$. Profiles of the isosurfaces of the interface $\{\phi=0\}$ are taken at $t=0,1.7,1.9,2.1,2.3$, and 2.9; (b) the experimental results by Brereton and Korotney in [5].

from the coaxial simulation is that their staggered position makes the two droplets become a hat shape with non-symmetrical structure. As they rise gradually, the lower droplet moves faster, while the upper droplet moves slowly, so the lower droplet will eventually be captured by the upper droplet ("fish-capture" phenomenon), and then the two droplets will be completely fuse together to form a large, obviously inclined cap-shaped drop. Similarly, we also compare with the experimental results given [5] shown in the Figure 9b. It can be seen that for the oblique coalescence case, our numerical simulation is also very consistent with the experimental results qualitatively.

\section{CONCLUding REMARKS}

The purpose of this paper is to construct a new fully discrete finite element numerical scheme for solving the Cahn-Hilliard-Navier-Stokes phase-field model with variable density and viscosity. With the help of multiple nonlocal variables and some specially designed ODEs, this scheme has the characteristics of linearity, full decoupling, and easy implementation. The calculation cost of each step only includes solving a few linear elliptic equations, and most of them are constant coefficients. We strictly show the solvability and energy stability and give the implementation process in detail. Various 2D and 3D numerical examples are performed to demonstrate the accuracy and efficiency. Some benchmark simulations, such as Rayleigh-Taylor instability and drop rising dynamics, are also carried out, and the good agreement with physical experiments proves the effectiveness of the scheme. 
Acknowledgements. The work of C. Chen was supported by the NSFC-11771375 and NSFC-11571297, Shandong Province Natural Science Foundation ZR2018MAQ008. The work of X. Yang was partially supported by National Science Foundation with grant numbers DMS-1818783 and DMS-2012490.

\section{REFERENCES}

[1] H. Abels, H. Garcke and G. Grün, Thermodynamically consistent, frame indifferent diffuse interface models for incompressible two-phase flows with different densities. Math. Models Methods Appl. Sci. 22 (2012) 1150013, 40.

[2] M. Alizadeh, S.M. Seyyedi, M. Taeibi Rahni and D.D. Ganji, Three-dimensional numerical simulation of rising bubbles in the presence of cylindrical obstacles, using lattice boltzmann method. J. Mol. Liq. 236 (2017) 151-161.

[3] D. Bhaga and M.E. Weber, Bubbles in viscous liquids: shapes, wakes and velocities. J. Fluid Mech. 105 (1981) 61-85.

[4] F. Boyer and S. Minjeaud, Numerical schemes for a three component Cahn-Hilliard model. ESAIM: M2AN 45 (2011) $697-738$.

[5] G. Brereton and D. Korotney, Coaxial and oblique coalescence of two rising bubbles, edited by I. Sahin and G. Tryggvason. In: Dynamics of Bubbles and Vortices Near a Free Surface, AMD-Vol. ASME, New York (1991).

[6] Y. Cai and J. Shen, Error estimates for a fully discretized scheme to a Cahn-Hilliard phase-field model for two-phase incompressible flows. Math. Comput. 87 (2018) 2057-2090.

[7] C. Chen and X. Yang, Efficient numerical scheme for a dendritic solidification phase field model with melt convection. J. Comput. Phys. 388 (2019) 41-62.

[8] C. Chen and X. Yang, Fast, provably unconditionally energy stable, and second-order accurate algorithms for the anisotropic Cahn-Hilliard Model. Comput. Meth. Appl. Mech. Eng. 351 (2019) 35-59.

[9] W. Chen, Y. Liu, C. Wang and S. Wise, Convergence analysis of a fully discrete finite difference scheme for cahn-hilliard-heleshaw equation. Math. Comput. 85 (2016) 2231-2257.

[10] A. Diegel, C. Wang, X. Wang and S. Wise, Convergence analysis and error estimates for a second order accurate finite element method for the Cahn-Hilliard-Navier-Stokes system. Numer. Math. 137 (2017) 495-534.

[11] H. Ding, P.D. M Spelt and C. Shu, Diffuse interface model for incompressible two-phase flows with large density ratios. J. Comput. Phys. 226 (2007) 2078-2095.

[12] Q. Du and R.A. Nicolaides, Numerical analysis of a continuum model of phase transition. SIAM J. Numer. Anal. 28 (1991) $1310-1322$.

[13] M. Gao and X.-P. Wang, An efficient scheme for a phase field model for the moving contact line problem with variable density and viscosity. J. Comput. Phys. 272 (2014) 704-718.

[14] V. Girault and P.A. Raviart, Finite Element Method for Navier-Stokes Equations: Theory and Algorithms. Springer-Verlag, Berlin, Heidelberg (1987) 395-414.

[15] H. Gomez, D.Z. Van and G. Kristoffer, Computational phase-field modeling, 2nd edition. In: Encyclopedia of Computational Mechanics. John Wiley \& Sons, Ltd. (2017).

[16] G. Grün and F. Klingbeil, Two-phase flow with mass density contrast: stable schemes for a thermodynamic consistent and frame-indifferent diffuse-interface model. J. Comput. Phys. 257 (2014) 708-725.

[17] J.L. Guermond and P. Minev, Higher-order time stepping for the incompressible Naveri-Stokes equations. SIAM. J. Sci. Comput. 37 (2015) A2656-A2681.

[18] J.L. Guermond and L. Quartapelle, A projection fem for variable density incompressible flows. J. Comput. Phys. 165 (2000) $167-188$.

[19] J.L. Guermond and A. Salgado, A splitting method for incompressible flows with variable density based on a pressure Poisson equation. J. Comput. Phys. 228 (2009) 2834-2846.

[20] J.L. Guermond and A.J. Salgado, Error analysis of a fractional time-stepping technique for incompressible flows with variable density. SIAM J. Numer. Anal. 49 (2011) 917-944.

[21] D. Han and X. Wang, A second order in time, uniquely solvable, unconditionally stable numerical scheme for Cahn-HilliardNavier-Stokes equation. J. Comput. Phys. 290 (2015) 139-156.

[22] D. Jacqmin, Diffuse interface model for incompressible two-phase flows with large density ratios. J. Comput. Phys. 155 (2007) 96-127.

[23] D. Li, Z. Qiao and T. Tang, Characterizing the stabilization size for semi-implicit fourier-spectral method to phase field equations. SIAM J. Numer. Anal. 54 (2016) 1653-1681.

[24] M. Li, Y. Cheng, J. Shen and X. Zhang, A bound-preserving high order scheme for variable density incompressible NavierStokes equations. J. Comput. Phys. 425 (2021) 109906.

[25] C. Liu, J. Shen and X. Yang, Decoupled energy stable schemes for a phase-field model of two-phase incompressible flows with variable density. J. Sci. Comput. 62 (2015) 601-622.

[26] Y. Liu, W. Chen, C. Wang and S. Wise, Error analysis of a mixed finite element method for a Cahn-Hilliard-Hele-Shaw system. Numer. Math. 135 (2017) 679-709.

[27] S. Minjeaud, An unconditionally stable uncoupled scheme for a triphasic Cahn-Hilliard/Navier-Stokes model. Numer. Methods Part. Differ. Equ. 29 (2013) 584-618.

[28] R. Nochetto and J.-H. Pyo, The Gauge-Uzawa finite element method part I: the Navier-Stokes equations. SIAM J. Numer. Anal. 43 (2005) 1043-1068. 
[29] J. Pyo and J. Shen, Gauge-Uzawa methods for incompressible flows with variable density. J. Comput. Phys. 221 (2007) 181-197.

[30] I. Romero, Thermodynamically consistent time stepping algorithms for nonlinear thermomechanical systems. Int. J. Numer. Meth. Eng. 79 (2009) 706-732.

[31] J. Shen and X. Yang, Numerical approximations of Allen-Cahn and Cahn-Hilliard equations. Disc. Cont. Dyn. Sys. A 28 (2010) 1669-1691.

[32] J. Shen and X. Yang, A phase-field model and its numerical approximation for two-phase incompressible flows with different densities and viscositites. SIAM J. Sci. Comput. 32 (2010) 1159-1179.

[33] J. Shen and X. Yang, Decoupled, energy stable schemes for phase-field models of two-phase incompressible flows. SIAM J. Num. Anal. 53 (2015) 279-296.

[34] G. Tryggvason, Numerical simulations of the Rayleigh-Taylor instability. J. Comput. Phys. 75 (1988) $253-282$.

[35] X. Yang, Linear, first and second order and unconditionally energy stable numerical schemes for the phase field model of homopolymer blends. J. Comput. Phys. 327 (2016) 294-316.

[36] X. Yang, A new efficient fully-decoupled and second-order time-accurate scheme for Cahn-Hilliard phase-field model of threephase incompressible flow. Comput. Methods Appl. Mech. Eng. 376 (2021) 13589.

[37] X. Yang. A novel fully-decoupled, second-order and energy stable numerical scheme of the conserved Allen-Cahn type flowcoupled binary surfactant model. Comput. Methods Appl. Mech. Eng. 373 (2021) 113502.

[38] X. Yang, A novel fully-decoupled, second-order time-accurate, unconditionally energy stable scheme for a flow-coupled volumeconserved phase-field elastic bending energy model. J. Comput. Phys. 432 (2021) 110015.

[39] X. Yang, Fully-discrete spectral-Galerkin scheme with decoupled structure and second-order time accuracy for the anisotropic phase-field dendritic crystal growth model. Int. J. Heat Mass Transfer 180 (2021) 121750.

[40] X. Yang, Numerical approximations of the Navier-Stokes equation coupled with volume-conserved multi-phase-field vesicles system: fully-decoupled, linear, unconditionally energy stable and second-order time-accurate numerical scheme. Comput. Methods Appl. Mech. Eng. 375 (2021) 113600.

[41] X. Yang, On a novel full decoupling, linear, second-order accurate, and unconditionally energy stable numerical scheme for the anisotropic phase-field dendritic crystal growth model. Int. J. Numer. Methods Eng. 122 (2021) 4129-4153.

[42] X. Yang and H. Yu, Efficient second order unconditionally stable schemes for a phase field moving contact line model using an invariant energy quadratization approach. SIAM J. Sci. Comput. 40 (2018) B889-B914.

[43] X. Yang and G.-D. Zhang, Convergence analysis for the invariant energy quadratization (IEQ) schemes for solving the CahnHilliard and Allen-Cahn equations with general nonlinear potential. J. Sci. Comput. 82 (2020) 55.

[44] H. Yu and X. Yang, Decoupled energy stable schemes for phase field model with contact lines and variable densities. J. Comput. Phys. 334 (2017) 665-686.

[45] P. Yue, J. Feng, C. Liu and J. Shen, A diffuse-interface method for simulating two-phase flows of complex fluids. J. Fluid Mech. 515 (2005) 293-317.

[46] G.-D. Zhang, X. He and X. Yang, Decoupled, linear, and unconditionally energy stable fully-discrete finite element numerical scheme for a two-phase ferrohydrodynamics model. SIAM J. Sci. Comput. 43 (2021) B167-B193.

\section{Subscribe to Open (S20) A fair and sustainable open access model}

This journal is currently published in open access under a Subscribe-to-Open model (S2O). S2O is a transformative model that aims to move subscription journals to open access. Open access is the free, immediate, online availability of research articles combined with the rights to use these articles fully in the digital environment. We are thankful to our subscribers and sponsors for making it possible to publish this journal in open access, free of charge for authors.

\section{Please help to maintain this journal in open access!}

Check that your library subscribes to the journal, or make a personal donation to the $\mathrm{S} 2 \mathrm{O}$ programme, by contacting subscribers@edpsciences.org

More information, including a list of sponsors and a financial transparency report, available at: https://www. edpsciences.org/en/maths-s2o-programme 